\title{
Connectomic Identification and Three-Dimensional Color Tuning of S-OFF Midget Ganglion Cells in the Primate Retina
}

\author{
는auren E. Wool, ${ }^{2}$ Orin S. Packer, ${ }^{1}$ QQ $a$ asim Zaidi, ${ }^{2}$ and Dennis M. Dacey ${ }^{1}$ \\ ${ }^{1}$ University of Washington, Department of Biological Structure, Seattle, Washington 98195, and ${ }^{2}$ State University of New York College of Optometry, \\ Graduate Center for Vision Research, New York, New York 10036
}

In the trichromatic primate retina, the "midget" retinal ganglion cell is the classical substrate for red-green color signaling, with a circuitry that enables antagonistic responses between long (L)- and medium (M)-wavelength-sensitive cone inputs. Previous physiological studies showed that some OFF midget ganglion cells may receive sparse input from short (S)-wavelength-sensitive cones, but the effect of S-cone inputs on the chromatic tuning properties of such cells has not been explored. Moreover, anatomical evidence for a synaptic pathway from $S$ cones to OFF midget ganglion cells through OFF midget bipolar cells remains ambiguous. In this study, we address both questions for the macaque monkey retina. First, we used serial block-face electron microscopy to show that every $S$ cone in the parafoveal retina synapses principally with a single OFF midget bipolar cell, which in turn forms a private-line connection with an OFF midget ganglion cell. Second, we used patch electrophysiology to characterize the chromatic tuning of OFF midget ganglion cells in the near peripheral retina that receive combined input from L, M, and S cones. These "S-OFF" midget cells have a characteristic S-cone spatial signature, but demonstrate heterogeneous color properties due to the variable strength of $\mathrm{L}, \mathrm{M}$, and $\mathrm{S}$ cone input across the receptive field. Together, these findings strongly support the hypothesis that the OFF midget pathway is the major conduit for S-OFF signals in primate retina and redefines the pathway as a chromatically complex substrate that encodes color signals beyond the classically recognized L versus $\mathrm{M}$ and $\mathrm{S}$ versus $\mathrm{L}+\mathrm{M}$ cardinal mechanisms.

Key words: color; connectome; midget pathway; primate; retina; vision

Significance Statement

The first step of color processing in the visual pathway of primates occurs when signals from short (S)-, middle (M)-, and long (L)-wavelength-sensitive cone types interact antagonistically within the retinal circuitry to create color-opponent pathways. The midget (L versus M or "red-green") and small bistratified (S vs L + M, or "blue-yellow") ganglion cell pathways appear to provide the physiological origin of the cardinal axes of human color vision. Here we confirm the presence of an additional S-OFF midget circuit in the macaque monkey fovea with scanning block-face electron microscopy and show physiologically that a subpopulation of S-OFF midget cells combine S, L, and M cone inputs along noncardinal directions of color space, expanding the retinal role in color coding.

\section{Introduction}

In humans and most nonhuman primates, color processing begins in the retinal circuitry where signals arising from three spec-

\footnotetext{
Received April 5, 2019; revised June 27, 2019; accepted Aug. 6, 2019.

Author contributions: L.E.W., O.S.P., Q.Z., and D.M.D. designed research; L.E.W., O.S.P., and D.M.D. performed research; L.E.W., Q.Z., and D.M.D. analyzed data; L.E.W. and D.M.D. wrote the paper.

This work was supported by National Institutes of Health (NIH) Grant RR-00166 to the Tissue Distribution Program of the National Primate Research Center at the University of Washington; and NIH Grants EY-07556 and EY-13312 (to Q.Z.), EY-06678 (to D.M.D.), and EY-01730 (to the Vision Research Core at the University of Washington). We thank Julian Vrieslander for programming assistance, and Beth Peterson, Ed Parker, Dale Cunningham, Yeon Jin Kim, and Ursula Bertram for their contributions. This study was undertaken at institutions occupying unceded Indigenous land of the Coast Salish people (specifically, the Duwamish Tribe; University of Washington) and the Lenape people (SUNY College of Optometry).

The authors declare no competing financial interests.

Correspondence should be addressed to Dennis M. Dacey at dmd@u.washington.edu.
}

trally distinct photoreceptor types (the long (L)-, middle (M)-, and short (S)-wavelength-sensitive cones) interact antagonistically. Two anatomically distinct circuits correlate strongly with cardinal directions in color space that have classically been identified in human psychophysics (Krauskopf et al., 1982): a "redgreen" mechanism, which combines antagonistic inputs from $\mathrm{L}$ and $\mathrm{M}$ cones and is served by the midget ganglion cell (Dacey and Lee, 1994; Crook et al., 2011); and a "blue-yellow" mechanism in which $\mathrm{S}$ cones oppose a combined $\mathrm{L}+\mathrm{M}$ cone signal and is served by the S-ON small bistratified ganglion cell (Dacey and Lee, 1994). The midget $L$ versus $M$ pathway includes ON and OFF cell

L.E. Wool's present address: Institute of Neurology, UCL, Gower Street, London WC1E 6BT, United Kingdom. https://doi.org/10.1523/JNEUROSCI.0778-19.2019

Copyright $\odot 2019$ the authors 
populations responding positively to increments and decrements, respectively. The S-ON pathway has been characterized in detail (Mariani, 1984; Kouyama and Marshak, 1992; Calkins et al., 1998; Herr et al., 2003; Crook et al., 2009), but a complementary S-OFF circuit for the transmission of S-cone decrements has remained elusive.

Two pieces of evidence point to an anatomically and physiologically distinct subpopulation of OFF-type midget ganglion cells as the origin of the S-OFF chromatic pathway (Dacey et al., 2014). First, elegant serial section electron microscopy (EM) from the macaque monkey fovea showed that OFF midget bipolar cells made basal, OFF-type contacts with presumed S-cones and would thus clearly comprise an S-OFF midget pathway (Klug et al., 2003). Second, midget ganglion cells in the retinal periphery, especially the OFF-type, can combine inputs of the same sign from S, L, and M cone types (Field et al., 2010).

In the greater scope of S-cone circuitry, the significance of these two results remains unclear. S-OFF cells (Valberg et al., 1986) recorded at the level of the lateral geniculate nucleus (LGN) show spatiotemporal properties that argue strongly against their basis in the midget pathway (Tailby et al., 2008a,b). More fundamentally, how-or even whether-additional weak $S$ input to peripheral midget cells affects their color tuning properties is unknown. Furthermore, the striking absence of an S-OFF midget pathway has been demonstrated in a marmoset (Lee et al., 2005), despite the clear identification of a homologous S-ON pathway circuitry in this New World monkey (Ghosh et al., 1997). The lack of an S-OFF midget pathway in marmoset echoes an earlier serial electron microscopic study in human retina that also failed to observe the presence of an OFF midget bipolar contact at the presumed S cone pedicle (Kolb et al., 1997). Correspondingly, the lack of a D-wave S-OFF signature in electroretinography (ERG) has been taken as evidence for the absence of an S-OFF pathway in the human retina (Maguire et al., 2018). Finally, it has been shown that a sign-inverting amacrine cell circuit (Chen and $\mathrm{Li}$, 2012) can be used to create an S-OFF pathway in a ground squirrel (Sher and DeVries, 2012). Thus, both the anatomical origins and functional properties of an S-OFF color opponent pathway remain unsettled.

Here we apply serial block-face electron microscopy to the macaque monkey fovea to show that all $\mathrm{S}$ cones synapse with a single OFF midget bipolar cell, which in turn makes a private-line connection with an OFF midget ganglion cell, confirming and extending the earlier result (Klug et al., 2003). Additionally, through single-cell patch recordings, we report OFF midget ganglion cells in the near retinal periphery that receive $S$ input and exhibit heterogeneous color properties due to combined inputs from L, M, and S cones. Our results advance the hypothesis that the OFF midget pathway is the principal conduit for S-OFF signals in macaque retina. Moreover, this work identifies a chromatically complex substrate at the retinal level that can encode color signals distinct from the cardinal $L$ versus $M$ and $S$ versus $L+M$ axes.

\section{Materials and Methods}

Retinal isolation. Eyes were removed from deeply anesthetized male and female macaque monkeys at the time of being killed (Macaca nemestrina, Macaca fascicularis, or Macaca mulatta) via the Tissue Distribution Program of the Washington National Primate Research Center and in accordance with protocols reviewed and approved by the University of Washington Institutional Animal Care and Use Committee.

After enucleation, the anterior chamber of the eye was removed, the vitreous drained, and the remaining eyecup placed in oxygenated Ames medium (A1420, Sigma-Aldrich). The choroid was then carefully dissected from the sclera to maintain intimate contact among neural retina, retinal pigment epithelium, and choroid.

Serial block-face electron microscopy. Tissue was first isolated as described above. After isolation, the central retina (including the fovea) was dissected and placed in a $4 \%$ glutaraldehyde solution for $\sim 2 \mathrm{~h}$ of fixation. The foveal tissue was then rinsed thoroughly in cacodylate buffer, $\mathrm{pH} 7.4$ $(0.1 \mathrm{M})$, and incubated in a $1.5 \%$ potassium ferrocyanide and $2 \%$ osmium tetroxide $(\mathrm{OsO} 4)$ solution in $0.1 \mathrm{~m}$ cacodylate buffer for $1 \mathrm{~h}$. After washing, the tissue was placed in a freshly made thiocarbohydrazide solution $\left(0.1 \mathrm{~g}\right.$ in $10 \mathrm{ml}$ double-distilled $\mathrm{H}_{2} \mathrm{O}$ heated to $60^{\circ} \mathrm{C}$ for $1 \mathrm{~h}$ ) for $20 \mathrm{~min}$ at room temperature (RT). After another rinse at RT, the tissue was incubated in $2 \% \mathrm{OsO} 4$ for $30 \mathrm{~min}$ at RT. The samples were rinsed again and stained en bloc in $1 \%$ uranyl acetate overnight at $40^{\circ} \mathrm{C}$, washed, and stained with Walton's lead aspartate for $30 \mathrm{~min}$. After a final wash, the retinal pieces were dehydrated in a graded alcohol series and placed in propylene oxide at RT for $10 \mathrm{~min}$. The tissue was then embedded in Durcupan resin (44610, Sigma-Aldrich). Semithin vertical sections through the retinal layers $(0.5-1 \mu \mathrm{m}$ thick) were cut and stained with toluidine blue and examined to determine the location of the foveal center. A region of interest was chosen on the foveal slope $\sim 400 \mu \mathrm{m}$ from the foveal center for block-face imaging in the scanning electron microscope (SEM). The block was then trimmed, gold-coated by standard methods, and mounted in a GATAN/Zeiss (3View) SEM. The block face was imaged in an array of $2540 \times 40 \mu \mathrm{m}$ tiles ( $10 \%$ overlap between tiles) that extended from the Henle fiber layer to the optic fiber layer $(\sim 200$ $\mu \mathrm{m}$ vertical and lateral extent; Fig. $1 A$ shows an image of most of the sampled area). The block face was imaged after each of 420 sections cut at $80 \mathrm{~nm}$ thickness. Scanning was performed with a $5 \mathrm{~nm} x y$ resolution and a dwell time of $1 \mu \mathrm{s}$. The resulting set of 10,500 TIFF images were contrast normalized, stitched into 420 layers, then aligned into a volume using procedures (align multilayer mosaic option) available with TrakEM2 software (Cardona et al., 2012; plug-in for NIH ImageJ, FIJI). In brief, for both within-layer and across-layer alignments, the expected transformation was set to Rigid to minimize scale change across layers, while the desired transformation was set to Affine to minimize alignment error. Residual alignment jitter was further reduced by applying an Affine regularizer. Cell and circuit reconstructions were performed using TrakEM2 to first create skeletons of cones, bipolar cells, and ganglion cells. Terminal nodes within these skeletons were placed on synaptic ribbons so that the $x, y, z$ coordinates and the number of synapses could be determined for a sample of cells. Volume rendering of selected cell profiles and ribbon content (Fig. 1B) was performed manually via inspection by multiple annotators.

In vitro preparation. After retinal isolation as described above, radial cuts were made in the isolated retina-choroid to create a flat mount that was adhered, ganglion-cell layer up, to the glass bottom of a thermostatically maintained $\left(\sim 36^{\circ} \mathrm{C}\right.$; TC-344B, Warner Instruments) steel superfusion chamber coated with poly-L-lysine $\left(10 \mathrm{mg}\right.$ in $10 \mathrm{ml} \mathrm{H}_{2} \mathrm{O}$; $\mathrm{P} 1399$, Sigma-Aldrich). The retina was continuously superfused with Ames' medium, pH 7.37 (constant oxygenation with $95 \% \mathrm{O}_{2} / 5 \% \mathrm{CO}_{2} ; 3-5 \mathrm{ml} /$ $\mathrm{min}$ ). Visual stimuli were projected onto the vitreal (ganglion-cell) side of the retina as in situ via the microscope objective lens, as described further below.

In vitro electrophysiology. Retinal ganglion cells were observed using a $60 \times$ water-immersion long-working distance objective (1.0 numerical aperture; Nikon) under infrared illumination. Using the "loose-patch" method, extracellular recordings were made with glass micropipettes (5-8 $\mathrm{M} \Omega$ ) filled with Ames' medium. When possible, the retinal location and distance from the foveal center of the recorded cells was determined and recorded as temporal equivalent eccentricity (Watanabe and Rodieck, 1989). To map the receptive field of the cell, the cell body was first placed in the middle of the stimulus field. Flashing white squares $(2 \mathrm{~Hz}$ temporal frequency, 10 or $25 \mu \mathrm{m}$ wide) were systemically moved in the $x$ and $y$ directions to locate the most sensitive point of the receptive field and determine its approximate size. The location of the maximum spike response was defined as the receptive-field midpoint; and visual stimuli were positioned relative to this point. Midget ganglion cells were visually identified and differentiated from other cell types by their relatively high 

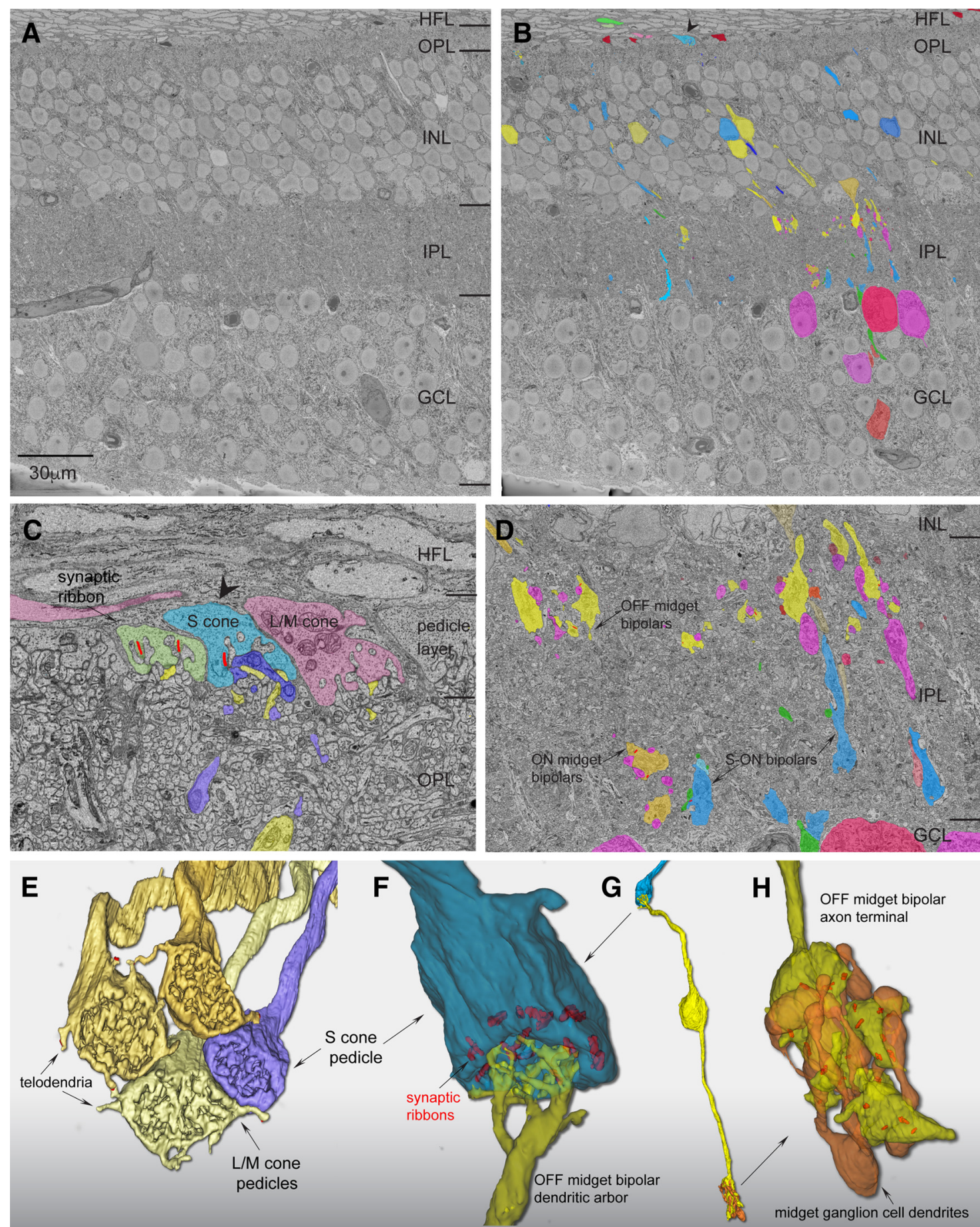

Figure 1. Scanning block-face electron microscopy demonstrates an S-OFF midget circuit in macaque retina. $\boldsymbol{A}$, Image of a single layer from the EM volume composed of a vertical section taken $400 \mu \mathrm{m}$ from the foveal center along the foveal slope. At this central location, ganglion-cell bodies stack up to six cell bodies thick. HFL, Henle fiber layer; OPL, outer plexiform layer; INL, inner nuclear layer; GCL, ganglion cell layer. $\boldsymbol{B}$, Profiles of volume-rendered processes in another vertical layer. In the OPL, OFF midget bipolar profiles are in yellow and blue cone (S ON) bipolar cells are in blue; at the top a pedicle identified as from an $S$ cone is shown in light blue (arrowhead) alongside a few scattered rod pedicles (dark red). Below in the INL are OFF midget bipolar cells (yellow) and blue cone (SON) bipolar cells (blue). In the GCL, some midget ganglion-cell bodies are shown in magenta and pink. C, Zoomed view of three pedicles in the OPL: the S cone in $\boldsymbol{B}$ (blue), flanked by two L/M cones (green and red). Blue cone (S ON) bipolar dendritic arbors (dark blue profile) form the invaginating central element and are encircled by the flat dendritic arbors (yellow profiles) of an OFF midget bipolar cell. D, Zoomed view of the IPL showing axons and axon terminals of OFF midget bipolar cells stratified near the outer border of the IPL (yellow profiles, arrow) and blue cone (S ON) bipolar cells stratified near the inner border of the IPL (blue profiles, arrows). A single ON midget bipolar terminal (orange profile; arrow) is also present. Midget ganglion-cell dendritic profiles in the $G C L$ are shown in magenta. $E$, Complete reconstruction of four neighboring cone pedicles from one $S$ cone and three $\mathrm{L} / \mathrm{M}$ cones. The $S$-cone pedicle is smaller than the $\mathrm{L} / \mathrm{M}$ cones and lacks the distinctive telodendria that connect adjacent L/M cones. $F$, Zoomed view of reconstructed S-cone pedicle in partial transparency, showing ribbon synapses (red) and the single dendritic arbor of an OFF midget bipolar cell in dense contact with the pedicle synaptic face. G, Zoomed out view of the entire reconstructed OFF midget bipolar circuit (yellow) linked to the S-cone pedicle (blue) shown in $\boldsymbol{F}$. The axon terminal exclusively contacts the compact dendritic arbor of an OFF midget ganglion cell (orange profile). $\boldsymbol{H}$, Zoomed view of the OFF midget bipolar axon terminal synaptic relation (yellow) with its associated OFF midget ganglion cell (orange) shown in partial transparency with synaptic ribbons (red). The ganglion cell for this particular private-line circuit was at the edge of the volume and could not be completely reconstructed. 
density and small soma size (Dacey and Lee, 1994), which was confirmed by receptive-field mapping: midget ganglion cells showed the smallest center diameters of any ganglion cell $(30-150 \mu \mathrm{m}$ in the near retinal periphery). The other relatively high-density ganglion-cell types (parasol and small bistratified cells) have relatively large cell bodies and thus show receptive field center diameters approximately two to three times larger than midget cells. Data acquisition and the delivery of visual stimuli were coordinated by custom software running on an Apple Macintosh computer. Current and spike waveforms were Bessel filtered at 2 or $5 \mathrm{kHz}$ and sampled at $10 \mathrm{kHz}$.

Stimulus generation. A digital light projector (Christie Digital Systems) was used to project the visual stimuli (VSG, Cambridge Research Systems) through an optical relay to the microscope camera port and to focus the image onto the photoreceptor layer. The irradiance spectra for red, green, and blue primaries were measured with a spectroradiometer (PR705, Photo Research); peak wavelengths and integrated photon fluxes were 636, 550, and $465 \mathrm{~nm}$ and $2.7 \times 10^{6}, 6.9 \times 10^{5}$, and $1.8 \times 10^{5}$ photons $/ \mathrm{s} / \mu \mathrm{m}^{2}$, respectively. To compute the effectiveness of the light delivered by each primary to the cone aperture, we calculated the products of each primary irradiance spectrum and each cone spectral sensitivity function (Baylor et al., 1987). We corrected for the spectrally broadening effects of self-screening by assuming a pigment density of $0.016 \mu \mathrm{m}$ (Baylor et al., 1987) and a cone outer segment length of $5 \mu \mathrm{m}$ : while cones at $\sim 8 \mathrm{~mm}$ have an outer segment length of $20 \mu \mathrm{m}$ (Banks et al., 1991), we correct for the fact that peripheral cones in vitro lay obliquely to the optical axis of the objective, thus shortening the effective path length and reducing light capture. Each product was then summed across wavelength giving units of "effective" photons per second per square micrometer (irradiance corrected by cone spectral sensitivity). Effective photons per second per square micrometer were then converted to photoisomerizations per second per cone by multiplying by the area of the cone aperture. In previous studies involving transverse illumination of the cone outer segment (Baylor et al., 1979), where funneling of the inner segments plays no role, the conversion factor commonly used is $0.37 \mu \mathrm{m}^{2}$. The efficiency of photoisomerization (0.67; Dartnall, 1972) is included in this value. In the in vitro macaque retina, as in the in vivo retina, light is incident upon the vitreal surface of the retina, and funneling by the inner segment would tend to increase the effective area of the cone aperture. We therefore consider the use of $0.37 \mu \mathrm{m}^{2}$ to be a very conservative estimate of cone aperture to make the conversion to photoisomerizations per second per cone.

Often, the intensity of the stimuli used in human visual psychophysics or in physiological experiments in the intact primate eye is expressed in units of retinal illuminance, or Trolands (Td). To aid comparison with our data, we previously calculated that for a peripheral cone with an inner segment aperture of $9 \mu \mathrm{m}, 1 \mathrm{Td}$ was equivalent to $\sim 30$ photoisomerizations/s/cone (Crook et al., 2009).

To generate stimuli that isolated responses from only L, M, or S cones, we computed the linear transformation between the measured primary irradiance spectra and the known spectral sensitivity functions for L, M, and S cones (Baylor et al., 1987). The solution to this transformation outputs the mixture of red, green, and blue primary intensities required to evoke isolated responses from L, M, or S cones (Brainard and Stockman, 2010). Our cone-isolating stimuli have been previously confirmed by direct recordings from macaque cones (Packer et al., 2010). Cone contrast was defined as the peak excursion from the background, divided by the mean light level, expressed as a percentage. Computed contrast for cone-isolating stimuli was $18 \%$ around a background comprising equal quantal catches for the three cone types. Additional high-contrast $\mathrm{S}$ and $\mathrm{L}+\mathrm{M}$ stimuli were $64 \%$ around an equal background.

Identifying putative S-cone input and characterizing cone-specific contributions to the receptive field. Retinal ganglion cells were initially surveyed for putative S-cone inputs by recording extracellular spike activity while presenting spots of high-contrast $\mathrm{S}$-cone or $\mathrm{L}+\mathrm{M}$-cone isolating stimuli to the receptive-field center of the cell (150 or $400 \mu$ m diameter; $64 \%$ contrast), modulated as square waves at $2 \mathrm{~Hz}$ temporal frequency. At this frequency, the $\mathrm{ON}$ response phase is $\sim 0^{\circ}$ and the OFF response phase is $\sim 180^{\circ}$. Stimulus presentations were repeated $12-20$ times, and peristimulus time histograms (PSTHs) were constructed from the spikes.
PSTHs were then fit with a Fourier series, and spiking activity was reported as the amplitude, $A$, and phase, $\theta$, of the first Fourier harmonic (F1). From this spiking activity, $\mathrm{S}$ or $\mathrm{L}+\mathrm{M}$ mechanism-specific responses were classified as either ON or OFF with strength $R=A \cos (\theta)$, where $-R$ denotes an OFF response and $+R$ denotes an $\mathrm{ON}$ response. While we always stimulated ganglion cells at photopic light levels to prevent against rod intrusion, this computation further penalizes any spurious rod response, which has a much longer latency to light increments $\left(\sim 270^{\circ}\right.$; Crook et al., 2009, their Fig. 5D). A minimum response threshold was determined by computing the response strength $R$ for a subset of midget ganglion cells recorded during epochs of no stimulus presentation $(1.00 \pm 0.62 \mathrm{spikes} / \mathrm{s} ; n=56)$ and discarding any cell with a response of $\leq 4$ SDs from the mean (i.e., 3.48 spikes/s). Cells with both $\left|R_{L+M}\right| \leq 3.5$ spikes/s and $\left|R_{S}\right| \leq 3.5$ spikes/s were considered unresponsive and were not included in further analyses. Midget cells with $\left|R_{S}\right|>3.5$ spikes/s were considered to have putative S-cone input.

To fully characterize the specific L-, M-, and S-cone inputs to individual cells, extracellular spike activity was recorded while presenting fullfield L-, M-, or S-cone isolating stimuli (diameter, 800 or $1200 \mu \mathrm{m} ; 18 \%$ contrast), modulated as square waves at a temporal frequency of $2 \mathrm{~Hz}$. Stimulus presentations were repeated 12-20 times; PSTHs were constructed and fit with a Fourier series, and spiking activity was reported as the $A$ and $\theta$ of the F1. From this spiking activity, cone-specific responses were classified as either ON or OFF with strength $R=A \cos (\theta)$, where $-R$ denotes an OFF response and $+R$ denotes an ON response. To compare the relative strength of L-, M-, and S-cone inputs to each cell, responses were normalized as $\overline{R_{L}}=R_{L} /\left(\left|R_{L}\right|+\left|R_{M}\right|+\left|R_{S}\right|\right), \overline{R_{M}}=$ $R_{M} /\left(\left|R_{L}\right|+\left|R_{M}\right|+\left|R_{S}\right|\right)$, and $\bar{R}_{S}=R_{S} /\left(\left|R_{L}\right|+\left|R_{M}\right|+\left|R_{S}\right|\right)$. For midget cells when the initial high-contrast $\mathrm{S}$ or $\mathrm{L}+\mathrm{M}$ stimulus was not run, we assessed responses to low-contrast stimuli with similar criteria to those described above to classify cells as unresponsive $\left(\left|R_{L}\right| \leq 3.5\right.$ spikes/s and $\left|R_{M}\right| \leq 3.5$ spikes/s).

Computing spatial frequency tuning. The spatial tuning of midget-cell receptive fields was characterized using $\mathrm{S}$ cone-isolating or $\mathrm{L}+\mathrm{M}$ coneisolating drifting gratings (modulated at $2 \mathrm{~Hz}$ temporal frequency) of varying spatial frequency [spatial frequency, 1/32-16 cycles per degree (cpd); contrast, $56 \%$ or $72 \%$ ]. For each cell, $A$ and $\theta$ were computed from the $\mathrm{F} 1$ at each stimulus frequency, and tuning curves were constructed from pairs of amplitude/phase measurements at each spatial frequency. Tuning curves were then fitted using a difference-of-Gaussians model (Enroth-Cugell et al., 1983) to determine the spatial arrangement of S and/or $\mathrm{L}+\mathrm{M}$ inputs to the receptive field center and surround. The details and application of this model have been described previously (Dacey et al., 2000; McMahon et al., 2004; Wool et al., 2018). From the fitted tuning curves, the frequency of peak response, $f_{\text {peak }}$, and the cutoff frequency, $f_{\text {cutoff }}$ (defined as the frequency at which response amplitude decreases to $1 / \sqrt{ } 2$ of the maximum) were computed for each cell for each stimulus. For a given cell, $f_{\text {cutoff }}>f_{\text {peak }}$ indicates that inputs are more localized to the smaller receptive-field center (i.e., tuned to higher spatial frequencies), while $f_{\text {cutoff }}<f_{\text {peak }}$ indicates that inputs are localized to the larger receptive-field surround (i.e., tuned to lower spatial frequencies).

Computing three-dimensional color tuning of ganglion cells. To measure the three-dimensional color tuning of cells (i.e., beyond $\mathrm{S}, \mathrm{L}-\mathrm{M}$, or $\mathrm{L}+\mathrm{M}$ ), we used a set of slow-modulating sinusoidal stimuli (Sun et al., 2006). These stimuli were constructed from a three-dimensional color space, $x y z$, defined by the following three classical postreceptoral mechanisms of LGN neurons: a cone-subtractive $(\mathrm{L}-\mathrm{M}) x$-axis, an $\mathrm{S}$ coneisolating $y$-axis, and a cone-additive $(\mathrm{L}+\mathrm{M}) z$-axis (Derrington et al., 1984). When the chromaticity of a full-field stimulus is modulated around circles in the three planes formed by these axes, it can be decomposed into the following three phase-shifted cone-isolating sinusoids: in the isoluminant $\mathrm{L}-\mathrm{M}$ versus $\mathrm{S}(x y)$ plane, $\mathrm{L}$ and $\mathrm{M}$ cones are in antiphase and are modulated $90^{\circ}$ out of phase with $\mathrm{S}$; in the $\mathrm{L}-\mathrm{M}$ versus $\mathrm{L}+\mathrm{M}(x z)$ plane, $\mathrm{L}$ and $\mathrm{M}$ are modulated $90^{\circ}$ out of phase with each other; and in the $\mathrm{S}$ versus $\mathrm{L}+\mathrm{M}(y z)$ plane, $\mathrm{L}$ and $\mathrm{M}$ are in phase but are $90^{\circ}$ out of phase with S. In any stimulus plane, vectors in the first and third quadrants represent additive input from the two mechanisms, whereas vectors in the second and fourth quadrants represent opponent inputs. The maximal cone contrast at the axes of the three stimulus planes was $8 \% \mathrm{~L}, 10 \%$ 
M, and 28\% S (xy); 13\% L, 14\% M, and 0\% S (xz); and 29\% L, 29\% M, $90 \% \mathrm{~S}(y z)$. The $x, y, z$ notation used in this study denotes generic Cartesian coordinates in three-dimensional space and should not be confused with the $\bar{x}, \bar{y}, \bar{z}$ notation used in the CIE (Commission Internationale de l'Eclairage) $1931 x, y, z$ color space (Schanda, 2007)—another common color space but one we do not use in this study.

Each stimulus was presented as a uniform field $(800-1200 \mu \mathrm{m})$ encompassing the receptive-field center and surround of the cell. Chromaticity was modulated at $1 \mathrm{~Hz}\left(360^{\circ} / \mathrm{s}\right.$, or $6^{\circ}$ per video frame $)$ in a clockwise (CW) and a counterclockwise (CCW) direction, averaged to prevent response latency from biasing the time (and, thus, angle) of maximum response. PSTHs (bin width $=12^{\circ}$ ) for both directions were constructed from the average spike rate across $12-24$ stimulus presentations. We performed Fourier analysis on each CW and CCW PSTH, and computed a response vector from the amplitude and phase of the F1. The preferred vector of the cell $(A, \theta)$ in a given plane was reported as the mean of the $\mathrm{CW}$ and CCW response vectors. To explore any clustering of preferred vectors in each stimulus plane, we used kernel density estimation to visualize the distributions of preferred vector angles, $\theta$ (preferred vector magnitude, $A$, was ignored), in response to each stimulus plane. For each cell type and stimulus plane, the distribution of preferred vector angles was binned (bin width $=1^{\circ}$ ), convolved with a Gaussian filter $\left(\sigma=10^{\circ}\right)$, and then normalized.

The strength of the responsiveness of the each cell to the $\mathrm{L}-\mathrm{M}(x), \mathrm{S}$ $(y)$, and L $+\mathrm{M}(z)$ chromatic mechanisms was determined by first decomposing its preferred vector $(A, \theta)$ in each stimulus plane $x y$ ('ISO'), $x z$ ('LvM'), and $y z$ ('SvLM') into two axis components ( $x$ and $y, x$ and $z$, or $y$ and $z$ ) using Equations 1-6:

$$
\begin{gathered}
x_{I S O}=A_{I S O} \cos \theta_{I S O} \\
y_{I S O}=A_{I S O} \sin \theta_{I S O} \\
x_{L v M}=A_{L v M} \sin \theta_{L v M} \\
z_{L v M}=A_{L v M} \cos \theta_{L v M} \\
y_{S v L M}=A_{S v L M} \cos \theta_{S v L M} \\
z_{S v L M}=A_{S v L M} \sin \theta_{S v L M}
\end{gathered}
$$

In these equations, $\theta_{I S O}, \theta_{L v M}$, and $\theta_{S v L M}$ are the preferred vector angles in the three planes, and $x, y$, and $z$ values are the weights of each component in each two-dimensional plane. For each pair of components in Equations 1-6, effective contrast along stimulus axes was equalized by correcting one component by the contrast ratio between axes [e.g., $\left.x_{I S O} \times\left(C_{y I S O} / C_{x I S O}\right)\right]$. Finally, to compute the $[X, Y, Z]$ vector projection in three-dimensional color space (where $x$ is the total contribution of the $\mathrm{L}-\mathrm{M}$ mechanism, $y$ is the total contribution of the $\mathrm{S}$ mechanism, and $z$ is the total contribution of the $\mathrm{L}+\mathrm{M}$ mechanism), each vector component was normalized by the total contrast of the stimulus plane, $C$, and preferred vector $A$. Since each component is doubly represented across the three total stimuli, the weights of each component were averaged over the number of component measurements, $n$. For a cell where all three stimulus planes were presented ( 146 cells), $n=2$ for each component, while for some cells where only two of the three total stimulus planes were presented ( 83 cells), $n=1$ for two of the three components. We found computing the weight of a component to be reliable regardless of one or two component measurements, as follows:

$$
\begin{gathered}
X=\frac{1}{n_{x}}\left[\frac{x_{I S O} A_{I S O}}{C_{I S O}}+\frac{x_{L v M} A_{L v M}}{C_{L v M}}\right] \\
Y=\frac{1}{n_{y}}\left[\frac{y_{I S O} A_{I S O}}{C_{I S O}}+\frac{y_{S v L M} A_{S v L M}}{C_{S v L M}}\right] \\
Z=\frac{1}{n_{z}}\left[\frac{z_{L v M} A_{L v M}}{C_{L v M}}+\frac{z_{S v L M} A_{S v L M}}{C_{S v L M}}\right] .
\end{gathered}
$$

$[X, Y, Z]$ coordinates were converted to spherical coordinates, and reported as the azimuth $\left(\varphi, 0-360^{\circ}\right.$ on the $x y$ isoluminant plane $)$ and absolute value of the elevation $\left(\theta, 0-90^{\circ}\right.$, where $0^{\circ}$ falls on the $x y$ plane and $90^{\circ}$ falls orthogonal to it, along the $z$-axis).

Statistics. All descriptive statistics denote mean \pm SD unless otherwise noted, and directional statistics are used to describe circular data (Berens, 2009). Two-sample Student's $t$ tests were used to compare L/M and S cone ribbon synapse numbers and to compare populations or distributions unless nonparametric data were assumed, in which case the twosample Kolmogorov-Smirnov test was applied. All analyses were completed in MATLAB R2015b (MathWorks).

\section{Results}

\section{Connectomic reconstruction of parafoveal S-cone OFF midget pathway}

We first identified putative $S$ cones by known differences in pedicle morphology. The pedicles of $\mathrm{S}$ cones differ qualitatively from neighboring $\mathrm{L}$ and $\mathrm{M}$ cones being smaller in size and lacking the short telodendritic processes present in $\mathrm{L}$ and $\mathrm{M}$ cones that create a gap junction-coupled network across $\mathrm{L}$ and $\mathrm{M}$ cones ( $\mathrm{O}$ 'Brien et al., 2012). Figure 1 illustrates a single layer in our foveal volume and the location of a single pedicle identified as an $S$ cone by these criteria (Fig. $1 B, C$, arrowhead). Figure $1 E$ shows a reconstruction of this pedicle (blue) in relation to three neighboring, larger pedicles (yellow shades); each of the larger pedicles gave rise to distinct telodendria that made tip-to-tip contacts with each other. By contrast, the smaller putative $S$ cone pedicle lacked telodendria and made no contacts with neighboring cone pedicles. This pedicle deployed 16 synaptic ribbons and was contacted by a single flat midget bipolar cell (Fig. $1 D-G$ ); this was reconstructed, and all synaptic ribbons were localized and their volumes rendered within the axon terminals (Fig. $1 G$ ). This midget bipolar terminal contained 24 synaptic ribbons, all of which were in exclusive contact with a single midget ganglion cell dendritic tree (Fig. $1 H$ ). If this cone and others showing the same unique morphology are indeed $\mathrm{S}$ cones, then they should form a selective ON pathway synaptic connection with the morphologically unique "blue cone" bipolar cell type (Kouyama and Marshak, 1992). Moreover, these S cones should form a regularly spaced array (Shapiro et al., 1985; Curcio et al., 1991; Bumsted and Hendrickson, 1999) and comprise $\sim 10 \%$ of the total number of cones in our volume.

There were 185 cone pedicles in the volume, and we classified 17 of these cones (9\%) as $\mathrm{S}$ cones based on their physical isolation from all larger neighboring cones; indeed, only these 17 pedicles lacked telodendria that contacted neighboring cones (Fig. 1E). There were also a number of rod spherules in our volume $(n=40$; Fig. $1 B$, top, dark red); consistent with a previous report, $\mathrm{S}$ cones-as well as L and M cones-gave rise to occasional telodendria that contacted the more outwardly situated rod spherules (O'Brien et al., 2012). These telodendria were situated at the outer edge of the pedicle layer in alignment with the rod spherule layer and above the synaptic face of the cone pedicles where coneto-cone telodendria are located. These pedicles also appeared to be dispersed regularly though the overall cone mosaic, as is expected for $\mathrm{S}$ cones (Fig. 2A).

We then reconstructed the bipolar cells linked to all invaginating central elements in each of these 17 cones. The result of this reconstruction (Fig. $2 B$ ) revealed that all central elements arose from a single-cone bipolar type with the well established morphology of the primate blue cone bipolar cell (Mariani, 1984; Kouyama and Marshak, 1992). We found 26 blue cone bipolar cells that formed divergent contacts exclusively to these 17 pedicles (1.5 bipolar cells per cone; Fig. $2 B$ ). A key identifying feature of this bipolar type is that it gives rise to several branching and occasionally long dendrites that bypass multiple cone pedicles to 


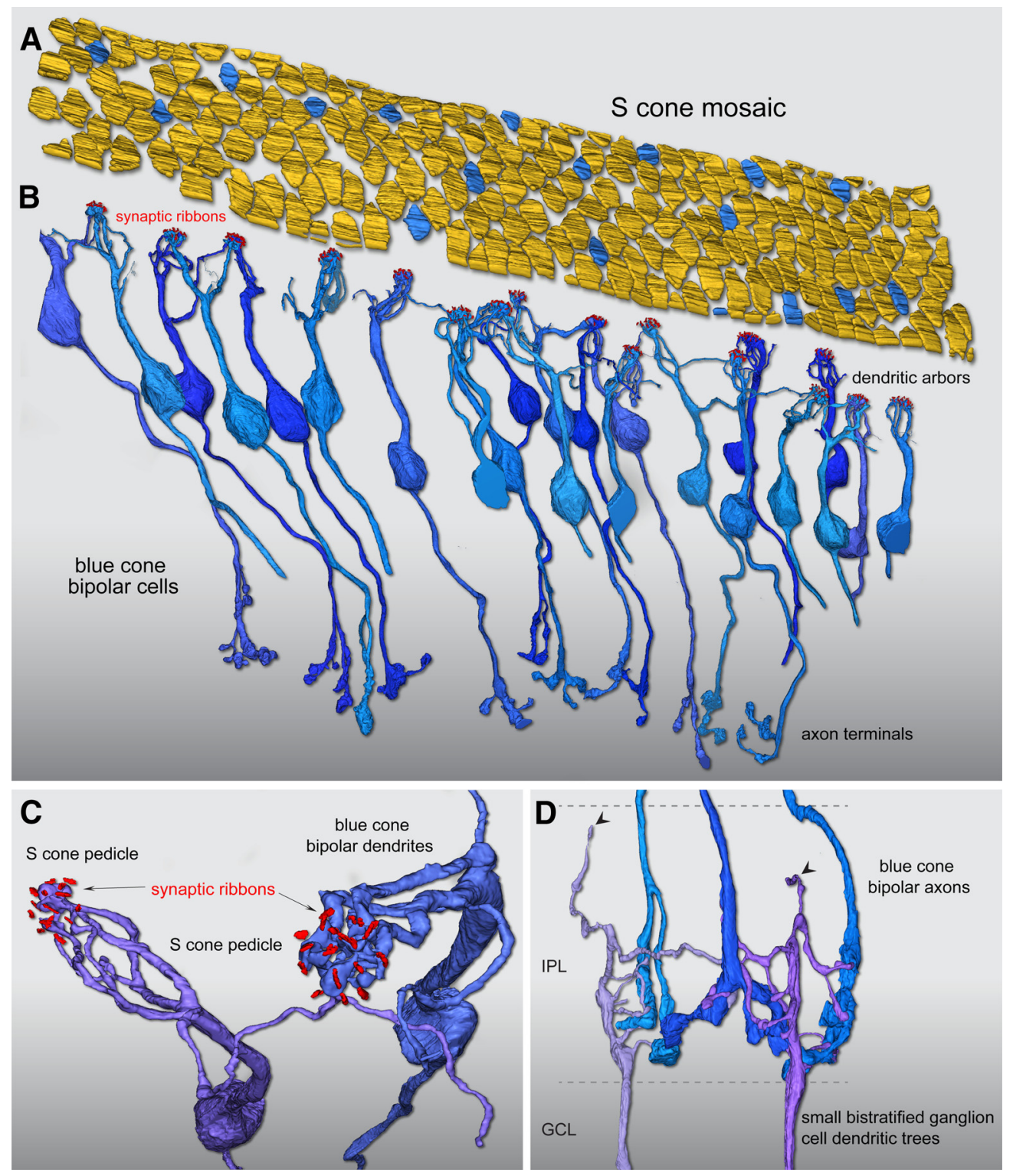

Figure 2. Reconstruction of foveal S-cone mosaic and blue cone (S ON) bipolar cell synaptic pathway. $\boldsymbol{A}$, Outlines of the positions of 185 cone pedicle locations in the foveal volume (Fig. 1). Seventeen regularly spaced cones (9\%) were identified as $\mathbf{S}$ cones (blue) by their small size and lack of telodendritic contacts with neighboring larger pedicles (Fig. 1). $\boldsymbol{B}$, Confirming the S-cone identity of these 17 pedicles, each received all invaginating central element contacts from a single cone bipolar type with the morphology of the blue cone (S ON) bipolar as previously described, with branching dendrites that converge on and selectively contact only S-cone pedicles, and may contact one to three neighboring S cones. In this instance, we found 26 blue cone (S ON) bipolar cells in synaptic contact with these $17 \mathrm{~S}$ cones, occupying all central elements in the pedicle. C, Zoomed view looking vertically through the pedicle of two blue cone (S ON) bipolar cell dendritic processes forming invaginating terminals (Fig. 1C) at neighboring S-cone pedicles. For visibility, the pedicle volume was made transparent; synaptic ribbon volumes (red) are shown in relation to the bipolar dendritic arbors. D, The axons of the blue cone (S ON) bipolar cells branch and terminate at the inner border of the IPL (Fig. 1D), where they make synaptic contact with presumed blue ON small bistratified ganglion cells. Two ganglion-cell dendritic trees receive synaptic input from three blue cone (SON) bipolar cell axon terminals at the inner border of the IPL. These two ganglion cells also show very sparse dendrites that extend to the outer half of the IPL (arrowheads), consistent with their identification as the blue ON small bistratified ganglion-cell type.

converge on one or two neighboring $\mathrm{S}$ cones (Fig. 2C; Kouyama and Marshak, 1992).

Of the 26 bipolar cells whose dendritic arbors were reconstructed, 12 cells were completely contained in our volume and could be reconstructed to their axon terminals and synaptic contacts with ganglion-cell dendrites in the inner plexiform layer (IPL; Fig. 2B,D). Again, as expected for the blue cone bipolar cell type, the axonal arbor was branched and terminated at or near the inner border of the IPL. In addition, these axon terminals did not contact midget ganglion cells but instead made converging synaptic connections with a larger multibranched dendritic tree that gave rise to occasional branches that extended to the outer, OFF subdivision of the IPL. This is consistent with previous findings that the blue-cone bipolar delivers ON-type $S$ cone input to the inner dendrites of the small bistratified blue ON ganglion-cell type (Dacey and Lee, 1994; Calkins et al., 1998; Crook et al., 2009), which displays a bistratified dendritic tree.

Each of these $17 \mathrm{~S}$ cones also received extensive noninvaginating or basal contacts (Figs. 1C,F, 3, 4A), many of which were large and encircled the invaginating central element in the triadassociated position (Calkins, 2001; Tsukamoto and Omi, 2015). These large contacts arose exclusively from a single OFF midget bipolar cell for each of the $17 \mathrm{~S}$ cones in the foveal volume (Fig. $3 A, B)$, which is identical to the pattern we observed for the first 


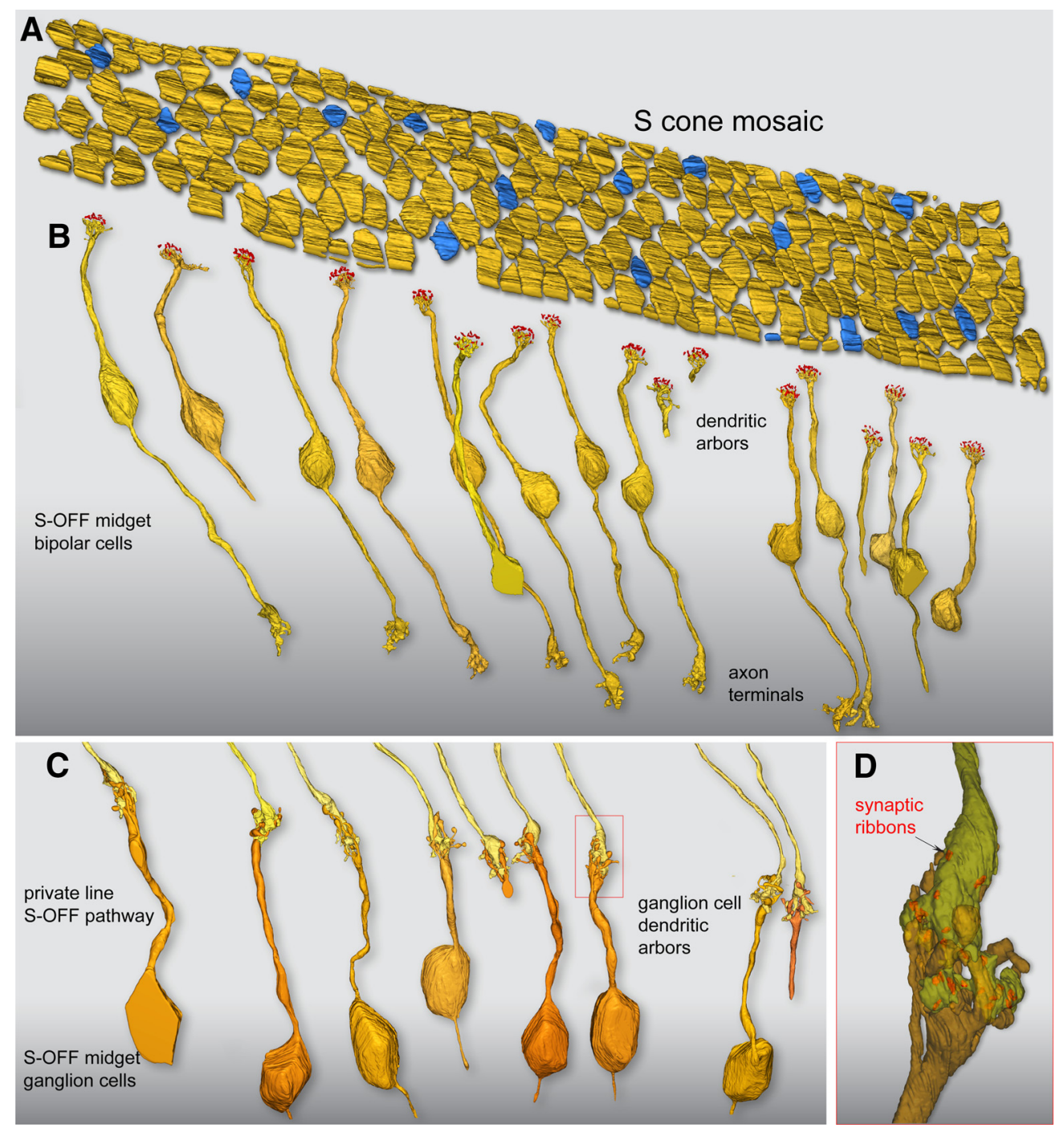

Figure 3. Flat contacts from midget bipolar cells form a private-line OFF pathway for $S$ cones. $A$, Outlines of pedicle positions with S-cone pedicles indicated in blue, as shown in Figure 2. $\boldsymbol{B}$, Each $\mathrm{S}$-cone pedicle is densely contacted by the noninvaginating, "flat" dendritic arbors of a single OFF midget bipolar cell. Of the $17 \mathrm{~S}-0 \mathrm{FF}$ midget bipolar dendritic arbors reconstructed, nine complete cells were encompassed within the volume and could be reconstructed completely to their axon terminals in the outer half of the IPL. C, Axon terminals of the nine complete 0FF midget bipolar cells shown in $\boldsymbol{B}$; each forms a private-line synaptic connection with the dendritic arbor of an OFF midget ganglion cell. Seven of the nine midget ganglion cells could be reconstructed to the cell body and axon, which extends into the optic fiber layer. $\boldsymbol{D}$, One of the OFF midget bipolar- ganglion cell private-line synapses in $\boldsymbol{C}$ (red box), shown in greater detail. The S-0FF midget bipolar-ganglion cell private-line synaptic complex is shown in partial transparency; the bipolar terminal enters from above (yellow) and the ganglion-cell dendritic arbor enters from below (orange). Twenty-five ribbon synapses in this bipolar cell were tagged and volume rendered (red), and established synaptic contact with this ganglion cell.

putatively identified S cone shown in Figure 1. These bipolar cells showed the distinctive midget bipolar dendritic and axonal morphology (Calkins et al., 1994): the cell body gave rise to a single stout dendrite that extended straight to the pedicle base before it arborized into a spray of short terminal branchlets at the synaptic face of the pedicle. This midget bipolar morphology contrasts with the relatively branched morphology of the blue cone bipolar cells (Figs. 2, 4B). Nine of these OFF midget bipolar cells could be reconstructed completely to their axon terminals in the outer portion of the IPL (Fig. 3C; see also Fig. 1D,H). The axon terminals formed a compact glomerular structure and private-line connection to an OFF midget ganglion cell classically described for foveal midget circuitry (Fig. 3D; Kolb and Dekorver, 1991; Calkins et al., 1994).

The OFF midget bipolar cells that were associated with $\mathrm{S}$ cones differed quantitatively from their $\mathrm{L} / \mathrm{M}$ cone counterparts in the number of synaptic ribbons present in the axon terminal (Figs. $3 D, 4 C)$. For the OFF midget bipolar cells connected to $S$ cones, the number of ribbon synapses $(23.9 \pm 3.2 ; n=9)$ was significantly lower than that found for a similar sample of neighboring OFF midget bipolar cells connected to $\mathrm{L}$ or $\mathrm{M}$ cones in the foveal volume (28.7 $\pm 3.9 ; n=9 ; p=0.012$, Student's $t$ test). Ribbon synapses were similarly reduced at the S-cone pedicle relative to the $\mathrm{L} / \mathrm{M}$-cone pedicles for these same cones, with S-cone pedicles showing $\sim 20 \%$ fewer synapses than their L/M-cone counterparts; this difference was also significant $(\mathrm{L} / \mathrm{M}$ cones $=21.67 \pm$ $1.00, n=9 ; \mathrm{S}$ cones $=16.89 \pm 1.7, n=9 ; p=0.0000018$, Student's $t$ test). The parallel reduction in ribbon synapse numbers in both the S-cone pedicle and the S-OFF midget bipolar terminal was not observed previously (Klug et al., 2003), and it was found that two $\mathrm{S}$-cone pedicles had a higher ribbon synapse number than the dominant $\mathrm{L}$ - and M-cone pedicles. Regardless, it does appear that the S-cone circuit can be identified by quantitative differences in synaptic architecture and that synaptic numbers in one part of the circuit can be mirrored at another point in the pathway. 

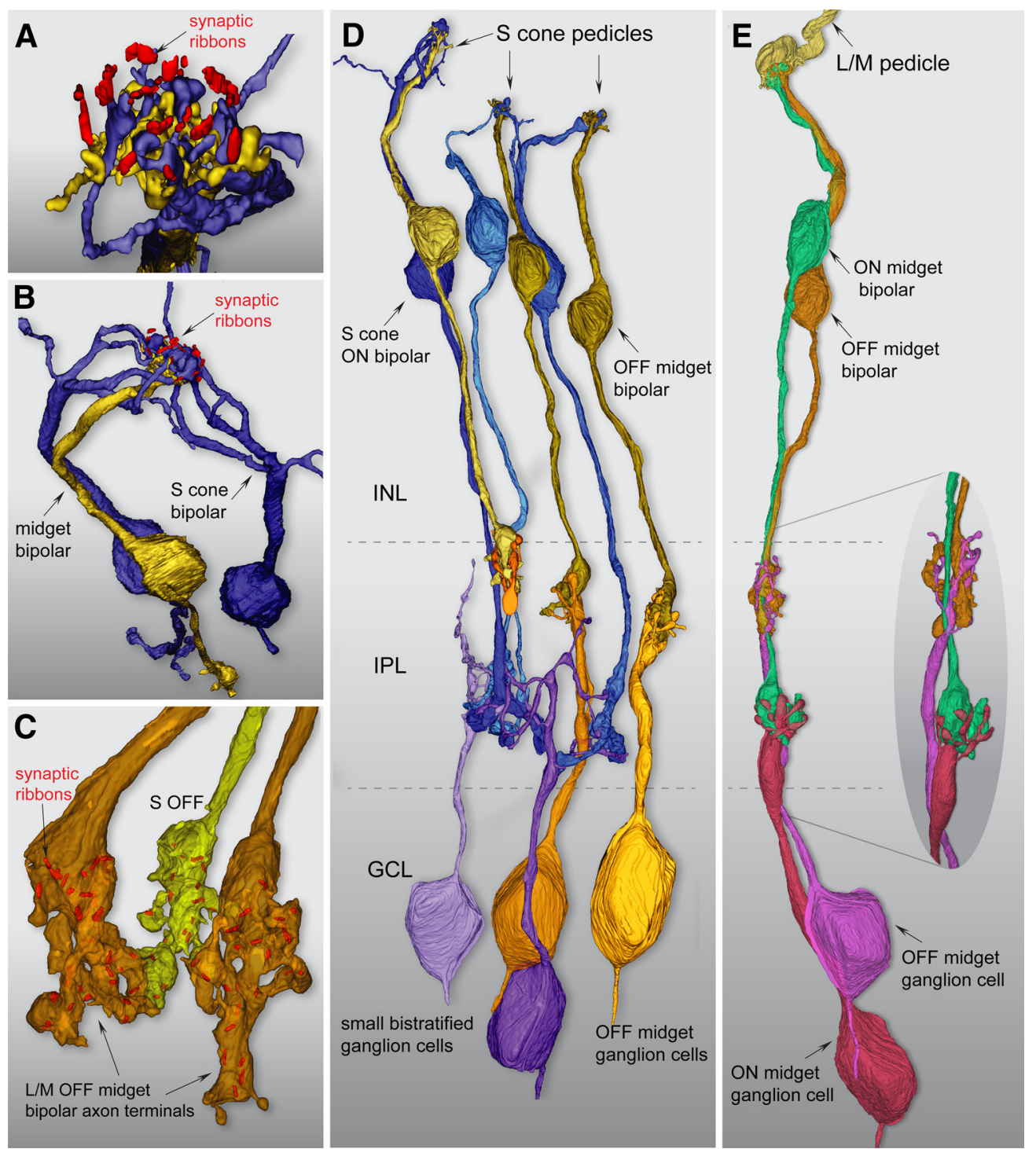

Figure 4. Summary of the morphological and synaptic asymmetry for ON and OFF S-cone pathways in macaque fovea. A, Zoomed view looking down through an S-cone pedicle (not shown) at invaginating blue cone (SON) bipolar (blue) and flat S-OFF midget bipolar (yellow) contacts, with the synaptic ribbon array (red). The blue cone (SON) bipolar cells give rise to very large terminations that occupy all central element positions in the pedicle, while the remaining fraction of the pedicle face is occupied by the flat contacts of a single OFF midget bipolar cell. $\boldsymbol{B}$, Another view looking down at a single S-cone ribbon array innervated by two branching and converging blue cone ( $\mathrm{ON}$ ) bipolar dendritic trees (blue). In contrast, a single private-line connection of an $0 \mathrm{FF}$ midget bipolar cell is present, in which a single stout dendrite forms a spray of terminal branches at the base of the pedicle (Fig. 1F). C, Two L/M OFF midget bipolar axon terminals (orange) and a single neighboring S-OFF midget bipolar terminal (yellow), with synaptic ribbons shown in red. S-OFF midget bipolar cell axon terminals show the typical midget morphology but exhibit fewer synaptic ribbons relative to their $\mathrm{L} / \mathrm{M}$ cone counterparts (see Results). $\boldsymbol{D}, \boldsymbol{E}$, Fundamental differences in $\mathrm{ON}$ and $\mathrm{OFF}$ circuitry originating from $\mathrm{S}$ cones and $\mathrm{L} / \mathrm{M}$ cones in foveal retina. $\boldsymbol{D}, 0 \mathrm{n}$ average, each S-cone pedicle synapses with 1.5 blue cone (SON) bipolar cells (blue) and 1 OFF midget bipolar cell (yellow). The blue cone (S ON) bipolar projects an axon terminal to the inner border of the IPL where it synapses with the blue $0 \mathrm{~N}$ small bistratified ganglion-cell type (purple shades), while the OFF midget bipolar forms a private-line synapse with an OFF midget ganglion cell (yellow shades). $\boldsymbol{E}$, Reconstruction of a complete L/M cone midget circuit. The L/M cone pedicle (yellow) synapses with a single $\mathrm{ON}$ (green) and OFF (orange) midget bipolar cell; these in turn form private-line synaptic complexes with an inner-stratifying $\mathrm{ON}$ (red) and outer-stratifying OFF (magenta) midget ganglion cell, respectively. The entire circuit is entwined in close physical proximity throughout its length (inset).

In sum, we identified $17 \mathrm{~S}$ cones in our volume for the first time based on their unique pedicle morphology; these 17 cones also showed the expected density and spatial distribution expected for $\mathrm{S}$ cones. In addition, the central elements for these cones were all occupied by an ON bipolar type with the distinctive dendritic and axon morphology of the previously described blue cone bipolar cell (Mariani, 1984; Boycott and Wässle, 1991; Kouyama and Marshak, 1992; Herr et al., 2003). This further confirms their identity as $\mathrm{S}$ cones and establishes the unique convergence of morphological features that identifies the $S$ cone circuitry in our foveal volume. We compared the connectivity observed at $\mathrm{S}$ cones to a sample of $\mathrm{L} / \mathrm{M}$ cones in our volume $(n=$
17) and observed that ON midget bipolar cells occupied most of the central element positions at the pedicle. Each ON midget bipolar cell formed a private-line circuit with its ON midget ganglion-cell partner and was tightly entwined with a private-line OFF midget bipolar circuit that mostly made triad-associated flat contacts with the pedicle (Fig. 4E), again as previously described (Kolb and Dekorver, 1991; Calkins et al., 1994). At the single-cell level of morphology, the L/M cone- and S cone-linked OFF midget circuits in this region appear identical. The presence of a private-line OFF midget bipolar-OFF midget ganglion cell connection associated with each of the $17 \mathrm{~S}$ cones clearly establishes this pathway as a major S-OFF pathway in the macaque monkey 

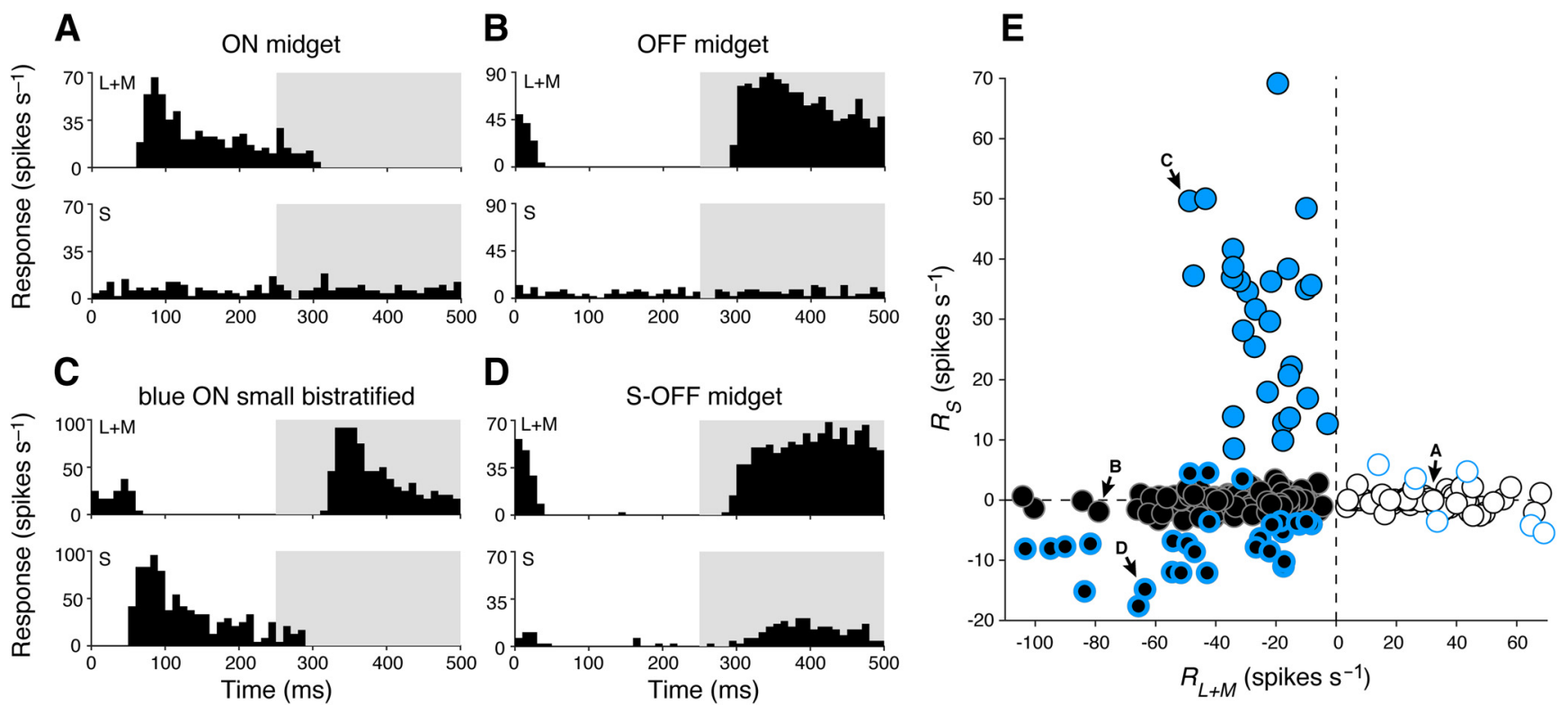

Figure 5. Cone-mechanism-specific responses in retinal ganglion-cell subtypes. $A$, Peristimulus time histogram of a typical $0 \mathrm{~N}$ midget ganglion cell shows spiking activity in the $0 \mathrm{~N}$ phase $(0-250$ $\mathrm{ms}$ ) of a $2 \mathrm{~Hz}$ modulating $(\mathrm{L}+\mathrm{M})$-cone-isolating stimulus (top) while showing no activity during a modulating $\mathrm{S}$-cone-isolating stimulus (bottom). $\boldsymbol{B}, \mathrm{A}$ typical $0 \mathrm{FF}$ midget cell shows the reverse behavior, with spiking activity in the $0 \mathrm{FF}$ phase $(250-500 \mathrm{~ms}$ ) of a $2 \mathrm{~Hz}$ modulating $\mathrm{L}+\mathrm{M}$ cone-isolating stimulus (top) but also showing no activity during a modulating S-cone-isolating stimulus (bottom). C, A blue $0 \mathrm{~N}$ ganglion cell responds to both $\mathrm{L}+\mathrm{M}$ (top) and $\mathrm{S}$ (bottom) cone-isolating stimuli, with opposite phase. $\boldsymbol{D}$, An S-OFF midget ganglion cell demonstrates classical sensitivity to $\mathrm{L}+\mathrm{M}$-cone stimuli (top), as well as a small response to $\mathrm{S}$-cone stimuli (bottom); both responses occur in the $0 \mathrm{FF}$ phase. $E$, Ganglion-cell types cluster based on their responsiveness to $S$-cone $\left(R_{S}\right)$ and $\mathrm{L}+\mathrm{M}$-cone $\left(R_{L+M}\right)$ stimulation. Blue circles, blue $0 \mathrm{~N}$ cells; white circles, $0 \mathrm{~N}$ midget cells; black circles, $0 \mathrm{FF}$ midget cells. Blue-bordered white and black circles denote midget ganglion cells with $\left|R_{S}\right|$ $>3.5$ spikes/s. Labeled cells are those shown in $\mathbf{A}-\boldsymbol{D}$, respectively.

foveal retina. We thus sought to use physiological methods to functionally characterize this S-OFF midget signal and determine its contribution to color tuning in midget ganglion cells outside of the foveal center where S, L, and $\mathrm{M}$ cone inputs converge within the receptive field.

\section{Physiological targeting of midget ganglion cells in the near periphery}

A main aim of this study was to amplify the impact of our structural characterization of an S-OFF midget circuit by also characterizing the functional properties of this circuit in vitro. As the midget pathway historically has been considered to accept inputs from only $\mathrm{L}$ and $\mathrm{M}$ cones, the S-cone connectivity that we observed in reconstructions of these cells should have a clear impact on how these cells respond in situ to complex visual stimulinamely, color stimuli that combine signals from L, M, and S cones. To explore this, we recorded extracellular spiking activity from 404 retinal ganglion cells ( 28 blue ON, 281 OFF midget, 95 ON midget) in 37 retinae from male and female monkeys ( $M$. nemestrina, M. fascicularis, or M. mulatta), targeting nearperipheral locations. As noted briefly in the Materials and Methods, midget ganglion cells in the macaque retina could be unequivocally identified by their distinctive soma size, high maintained discharge rates, sustained light responses, and very small receptive-field center sizes (30-150 $\mu \mathrm{m}$ diameter) relative to all other ganglion cells; this is consistent with their very small dendritic tree diameters (Dacey and Petersen, 1992; Dacey, 1993; Diller et al., 2004; Crook et al., 2011; Wool et al., 2018). Parasol ganglion cells display large cell body diameters, transient light responses, and receptive fields approximately three times the diameter of midget cells. All other ganglion cell types exhibit receptive fields much larger than that of parasol cells (Crook et al., 2008). The distinction between midget ganglion cells and blue $\mathrm{ON}$ cells is also clear due to the distinctive response of the blue
ON cells to S-cone stimuli and their large receptive field size (Crook et al., 2009), as considered further below. As inputs to midget ganglion cells increase as a function of eccentricity (Wool et al., 2018), cells in our recording locations sample anywhere from 3 to 12 cones, theoretically maximizing the likelihood of encountering an S cone in the receptive field of a cell. Receptivefield mapping (see Materials and Methods) was used to differentiate midget ganglion cells from their two to three times larger blue ON counterparts. We then used a range of stimuli to characterize the cone-specific inputs, spatial properties, and chromatic signature of each cell. While our aim was to record the responses of all cells to the full set of stimuli, this was not always possible; thus, partial characterizations are also reported and the number of cells recorded during each stimulus is indicated. When possible, we recorded the retinal location of each cell in units of temporal equivalent eccentricity. Cells were located in the range of $9-48^{\circ}\left(30 \pm 7^{\circ} ; n=331\right)$.

\section{Some OFF midget ganglion cells respond to S-cone stimulation}

We delivered high-contrast S-cone- or $\mathrm{L}+\mathrm{M}$-cone-isolating stimuli (spots of 150 or $400 \mu \mathrm{m}$ diameter, $64 \%$ contrast) to the receptive-field center of each cell. While initial receptive-field mapping easily distinguished blue $\mathrm{ON}$ cells from midget ganglion cells due to their relatively larger receptive-field size, blue $\mathrm{ON}$ cells were moreover characterized by a vigorous $\mathrm{ON}$ response to $\mathrm{S}$-cone stimulation. To these square-wave stimuli, responses to S-cone stimulation were entirely absent in most ON and OFF midget cells, and these cells were instead more reliably characterized by the phase of their response to $\mathrm{L}+\mathrm{M}$ stimulation: $\mathrm{ON}$ cells responded in the $\mathrm{ON}$ phase $\left(0-250 \mathrm{~ms}\right.$, or $\left.\sim 0^{\circ}\right)$ of the stimulus (Fig. 5A), while OFF cells responded in the OFF phase (250-500 $\mathrm{ms}$, or $\sim 180^{\circ}$; Fig. $5 B$ ). In comparison, all blue ON cells responded to $\mathrm{S}$-cone stimulation in the $\mathrm{ON}$ phase, and responded to $\mathrm{L}+\mathrm{M}$-cone stimulation in the OFF phase (Fig. $5 C$ ). Notably, in 
A
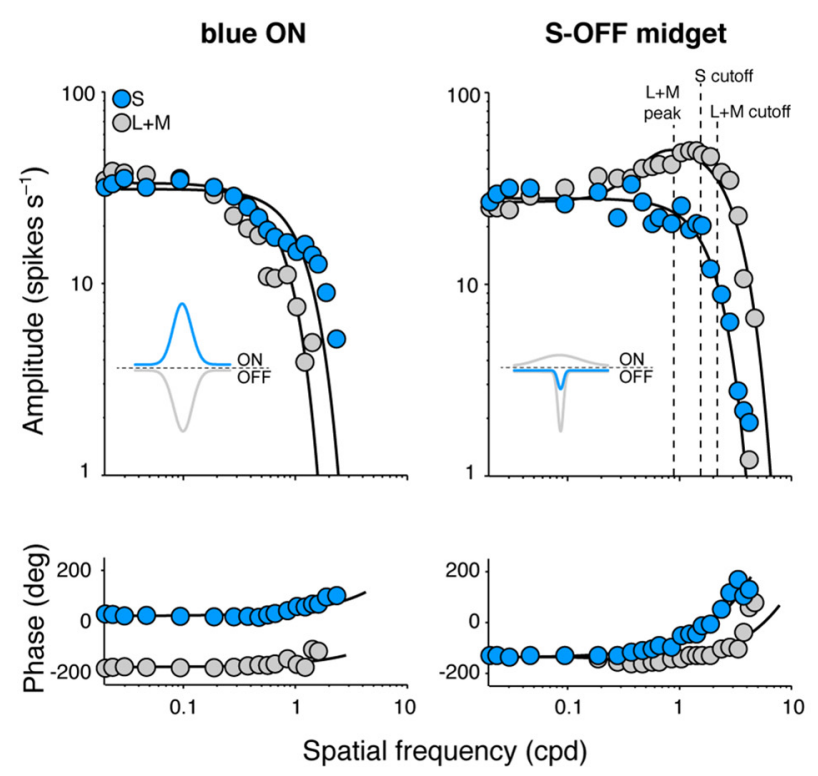

C

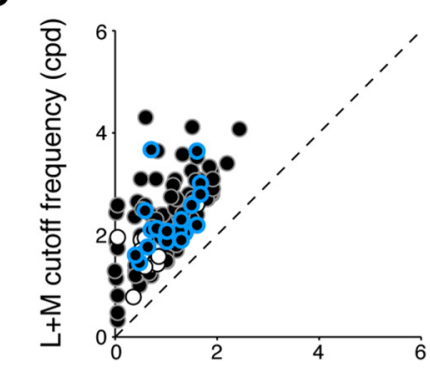

E
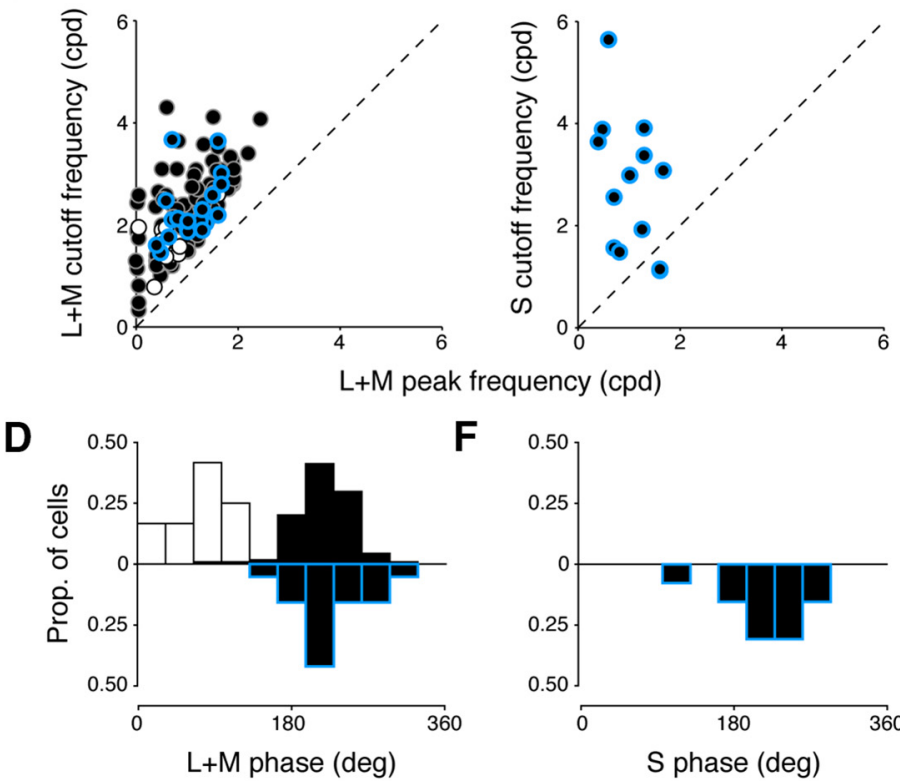

$\mathbf{F}$

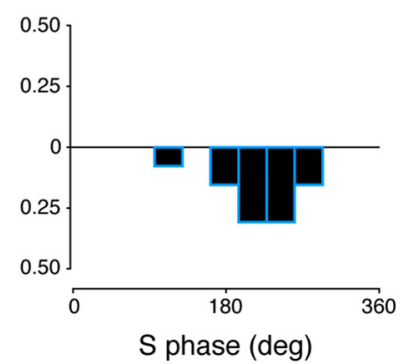

Figure 6. Spatial extent of S-cone input to OFF midget ganglion cells. $A, B$, Compared with blue ON ganglion cells, which demonstrate spatially coextensive, low-pass, and antiphase spatial tuning to both S- and L + M-cone gratings $(A)$, S-OFF midget ganglion cells demonstrate a low-pass spatial sensitivity to $S$-cone gratings atop the typical band-pass sensitivity to $L+M$-cone gratings, which occurs in the same phase $(\boldsymbol{B})$. Inset, Gaussian curves illustrate the type of receptive-field profile expected from each cell. $\boldsymbol{C}$, All midget ganglion cells demonstrate characteristic band-pass tuning to $L+M$ gratings, in which the cutoff frequency for $L+M$ gratings occurs at a higher spatial frequency than the peak frequency. The $L+M$ spatial profiles of putative $S-0 F F$ midget ganglion cells (black/blue circles) are compared with typical ON (white circles) and OFF (black circles) midget cells, with the cutoff frequency for $L+M$ gratings ( $y$-axis) plotted as a function of peak frequency ( $x$-axis). D, Distribution of response phase to L + M gratings for S-OFF (black/blue bars), ON (white bars), and OFF (black bars) midget cells. $E$, For S-OFF midget cells, cutoff frequency for $S$ gratings $(y$-axis) is plotted as a function of peak frequency for $L+M$ gratings ( $x$-axis). $F$, Distribution of response phase to $S$ gratings, for S-OFF midget cells.

a small subset of OFF midget cells, the characteristic OFF response to $\mathrm{L}+\mathrm{M}$-cone stimulation was accompanied by a small response to $\mathrm{S}$-cone stimulation in the same phase (Fig. 5D).

To compare the relationship of S- and $\mathrm{L}+\mathrm{M}$-cone response properties of blue $\mathrm{ON}$, ON midget, and OFF midget ganglion cells (including any midget ganglion cells with putative S-cone input), component-mechanism-specific responses $R_{S}$ and $R_{L+M}$ were computed from the $A$ and $\theta$ of spike activity during $S$ and $\mathrm{L}+\mathrm{M}$ stimulation (see Materials and Methods) to determine the relative strength of each mechanism. Blue ON, OFF midget, and ON midget cells form distinct clusters based on their relative responsiveness to $\mathrm{S}$ - or $\mathrm{L}+\mathrm{M}$-cone stimulation (Fig. 5E). ON and OFF midget cells are well defined by the sign of response to $\mathrm{L}+\mathrm{M}$ stimulation $\left(\mathrm{OFF}, R_{\mathrm{L}+\mathrm{M}}=-28.4 \pm 16.9\right.$ spikes/s; ON, $R_{\mathrm{L}+\mathrm{M}}=$ $24.9 \pm 16.1$ spikes/s), and the lack of response of most cells to $\mathrm{S}$-cone stimulation caused clustering at the $x$-axis $\left(\mathrm{OFF}, R_{S}=\right.$ $-1.1 \pm 0.90$ spikes/s; ON: $R_{S}=0.51 \pm 0.83$ spikes/s). Blue ON cells showed balanced and opponent S-ON and (L+M)-OFF responses $\left(R_{S}=30.5 \pm 14.5\right.$ spikes $/ \mathrm{s} ; R_{L+M}=-24.0 \pm 12.0$ spikes/s). While most $\mathrm{ON}$ and OFF midget cells showed no $\mathrm{S}$-cone response, the small subpopulation that did (OFF cells, in particular) fall farther off the $x$-axis. We classified as putative $\mathrm{S}$-cone midgets those cells with $\left|R_{S}\right|>3.5$ spikes/s, which was $<4$ SDs above the mean $R$ for a subset of cells where responses were recorded during epochs of no stimulus presentation $(1.00 \pm 0.62, n=56)$. This criterion identified a subset of 35 S-cone midget cells (29 OFF midgets, 6 ON midgets). For the majority of cells in this subset (26 OFF midgets, $3 \mathrm{ON}$ midgets), responses to $\mathrm{S}$-cone stimulation occurred in the same phase as responses to $\mathrm{L}+\mathrm{M}$ stimulation $\left(\mathrm{OFF}: R_{S}=\right.$ $-8.2 \pm 4.0 \mathrm{spikes} / \mathrm{s}, R_{L+M}=-43.2 \pm 29.1 \mathrm{spikes} / \mathrm{s} ; \mathrm{ON}: R_{S}=$ $4.7 \pm 1.1$ spikes/s, $R_{L+M}=28.2 \pm 14.8$ spikes/s). The remain- ing cells (three OFF midgets, three ON midgets) showed $\mathrm{S}$-cone responses in the opposite phase.

Spatial tuning of S-OFF midget cells identifies S-cone input to receptive-field centers

We next sought to identify the arrangement of S-cone input to the receptive fields of S-OFF midget cells. All ON and OFF midget ganglion cells exhibit classical center-surround receptive-field structure, due to a small central $\mathrm{ON}$ - or OFF-responsive region (formed by excitatory inputs from midget bipolar cells), surrounded by a broader region of opposite-phase sensitivity (formed by inhibitory inputs from horizontal cells; Crook et al., 2011). Thus, the OFF-phase S-cone response we observed in OFF-center midget cells is consistent with an anatomical arrangement where S-cone input is propagated by an OFF midget bipolar cell that contributes to the receptive-field center. We further explored this hypothesis by characterizing the spatial properties of cells to understand whether S-cone input was localized to the smaller receptive-field center (i.e., sensitive to higher spatial frequencies) or to the larger receptive-field surround (i.e., sensitive to lower spatial frequencies). For 145 midget ganglion cells (114 OFF midgets, 12 ON midgets, 19 S-OFF midgets), we measured spike discharges to $\mathrm{L}+\mathrm{M}$-cone and/or S-cone drifting gratings of increasing spatial frequency. While the sensitivity of $\mathrm{S}$-OFF cells to S-cone stimulation encourages an initial comparison to blue $\mathrm{ON}$ cells, the more well-known substrate of the retina for S-cone signals, we found the spatial tuning of S-OFF midgets to be qualitatively different from the spatial tuning of blue $\mathrm{ON}$ cells, suggesting a functional asymmetry in how S-cone signals are transmitted across the two ganglion-cell subclasses (Fig. 6). While blue ON cells show low-pass tuning to both L+M- and $\mathrm{S}$-cone gratings with a clear antiphase relationship between 
A

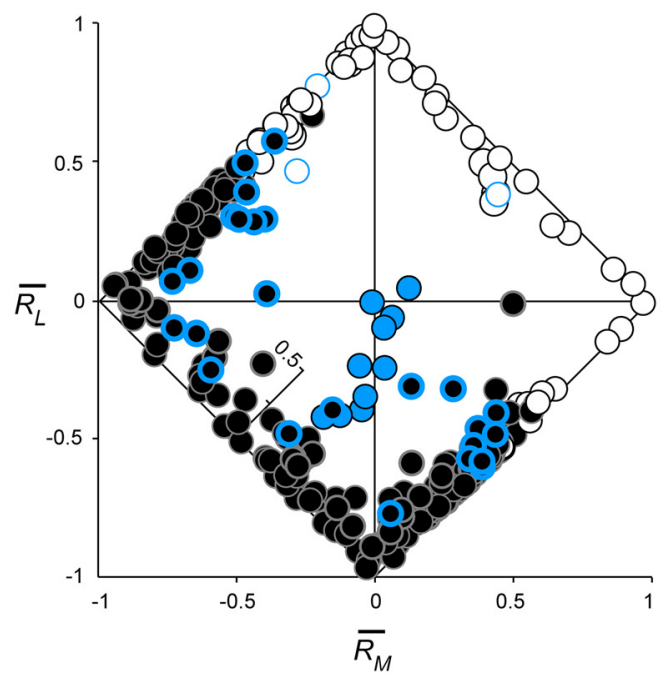

B

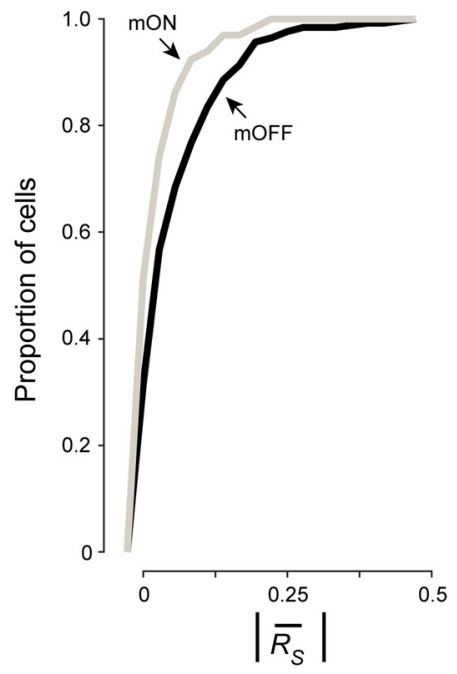

Figure 7. $\mathrm{L}-, \mathrm{M}$-, and $\mathrm{S}$-cone-specific inputs to ganglion-cell subtypes. $A$, Cone-specific responses $\overline{R_{L}} \overline{R_{M}}$, and $\overline{R_{S}}$ are shown for each ganglion cell by plotting relative $L$ - versus $M$-cone input on the $x$ - and $y$-axes, respectively. $S$-cone input is denoted by a third internal z-axis. Classical $L-M$ opponency is assessed by comparing the sign of $\overline{R_{L}}$ and $\left.\mathrm{i}\right] \overline{\mathrm{R}_{M}}$ : opponent cells fall along the diagonals where the signs of $\overline{R_{L}}$ and $\overline{R_{M}}$ are opposed, while nonopponent cells fall along the diagonals where the signs are matched. Blue $0 \mathrm{~N}$ cells (blue circles) demonstrate sign-matched L- and M-cone inputs, along with strong S-cone input. ON (white circles) and OFF (black circles) midget cells demonstrate both opponent and nonopponent L- and M-cone inputs, with OFF midget cells demonstrating stronger $S$-cone input overall. Blue-bordered white and black circles denote $0 \mathrm{~N}$ and OFF midget cells initially identified as S cone-sensitive in Figure 1. $\boldsymbol{B}$, Cumulative distribution of proportional S-cone input to OFF midget ganglion cells (black) compared with $0 \mathrm{~N}$ midget ganglion cells (gray).

mechanisms (Fig. 6A) due to spatially coextensive receptive fields (Crook et al., 2009), the responses of S-OFF midget cells showed different spatial tuning and phase properties during $\mathrm{L}+\mathrm{M}$ - and S-cone gratings (Fig. $6 B$ ), with a spatial profile much more similar to other OFF midget cells.

In response to $\mathrm{L}+\mathrm{M}$ drifting gratings, all $\mathrm{OFF}, \mathrm{ON}$, and $\mathrm{S}-\mathrm{OFF}$ midget cells showed characteristic band-pass tuning (diminished and antagonistic center-surround responses at low spatial frequencies, and enhanced center-isolating responses at high spatial frequencies; Fig. $6 B$ ). As such, there was a clear $f_{\text {peak }}$ for each cell and $f_{\text {cutoff }}>f_{\text {peak }}$ for all cells (Fig. 6C). For OFF midget cells without S-cone input, $f_{\text {peak }}=1.02 \pm 0.56 \mathrm{cpd}$ and $f_{\text {cutoff }}=2.23 \pm$ $0.75 \mathrm{cpd}$. For ON midget cells, $f_{\text {peak }}=0.65 \pm 0.37 \mathrm{cpd}$ and $f_{\text {cutoff }}=$ $1.65 \pm 0.44 \mathrm{cpd}$. The slightly lower spatial tuning of ON midget cells has been previously described, and is attributed to slightly larger dendritic trees (Watanabe and Rodieck, 1989; Dacey and Petersen, 1992; Dacey, 1993). S-OFF midget cells showed a similar spatial profile to other OFF midget cells in response to $\mathrm{L}+\mathrm{M}$ drifting gratings, with $f_{\text {peak }}=1.08 \pm 0.43 \mathrm{cpd}$ and $f_{\text {cutoff }}=2.30 \pm$ $0.61 \mathrm{cpd}$. Neither $f_{\text {peak }}$ nor $f_{\text {cutoff }}$ was statistically significantly different between OFF midget cells with or without S-cone input ( $p=0.65$ and $p=0.73$, respectively; Student's $t$ test). As expected, the phase of response to $\mathrm{L}+\mathrm{M}$ drifting gratings between $\mathrm{ON}$ and OFF midget cells differed by $\sim 180^{\circ}\left(\mathrm{ON}=75.5 \pm 34.8^{\circ}\right.$, $\left.\mathrm{OFF}=216.2 \pm 33.2^{\circ}\right)$. In S-OFF midget cells, we found that the phase of response to $\mathrm{L}+\mathrm{M}$ drifting gratings $\left(224.8 \pm 40.9^{\circ}\right)$ closely matched that observed in the other OFF midget cells; no statistically significant difference of response phase was found between the two populations ( $p=0.31$; Student's $t$ test; Fig. $6 D$ ).

While the spatial tuning and phase of $\mathrm{L}+\mathrm{M}$ inputs to S-OFF midget cells closely resemble those of typical OFF midget cells, we also characterized the spatial tuning and phase properties of their S-cone sensitivity using S-cone drifting gratings (14 S-OFF cells).
In contrast to the band-pass tuning curves observed for $\mathrm{L}+\mathrm{M}$ drifting gratings, responses to S-cone drifting gratings showed low-pass spatial tuning and were thus best fit with a single Gaussian function. The cutoff frequency of most S-OFF cells during S-cone stimulation $\left(f_{\text {cutoff }}=2.79 \pm 1.33\right.$ cpd) was greater than the peak frequency as identified during $\mathrm{L}+\mathrm{M}$ stimulation (Fig. $6 E)$; this high spatial frequency tuning suggests an arrangement of S-cone input sequestered to the receptive-field center. This is further supported by the phase of response to S-cone drifting gratings we observed in these cells $\left(218.5 \pm 43.9^{\circ}\right)$, which closely matches the response phase of both $\mathrm{S}-\mathrm{OFF}$ and OFF midget cells during the presentation of $\mathrm{L}+\mathrm{M}$ drifting gratings. This S-cone spatial profile of S-OFF cells that we describe-low-pass tuning, high $f_{\text {cutoff, }}$, and a response phase similar to other OFF cells-suggests that the S-cone contribution we observed in these cells is localized to the receptive field center, with a very weak and/or infrequent contribution from the receptive-field surround. This is consistent with the bipolar cellmediated S-cone connectivity we observed anatomically, which predicts functional inputs localized in this way.

$S$ cones contribute to both cone-opponent and nonopponent OFF midget cells

After identifying a subgroup of S-OFF midget cells and characterizing the spatial properties of their S-cone input, we then asked how this input was related to the classical $\mathrm{L}-\mathrm{M}$ color opponency observed in the midget pathway. We thus sought to determine whether the S-cone response had any systematic relation to the sign of either the $\mathrm{L}$ or $\mathrm{M}$ cone input to the receptive field. To answer this, we recorded extracellular spikes from 332 ganglion cells ( 10 blue ON cells, 256 OFF midget cells, 66 ON midget cells) during full-field L-, M-, and S-cone-isolating stimulation with square-wave stimuli ( $18 \%$ contrast, 800 or $1200 \mu \mathrm{m}$ in diameter), then computed the component mechanism-specific responses $R_{L}, R_{M}$, and $R_{S}$ from the F1 $A$ and $\theta$ values of each cell. To compare the proportional strength of L-, M-, and S-cone inputs to each cell, responses were normalized as $\overline{R_{L}}=R_{L} /\left(\left|R_{L}\right|+\left|R_{M}\right|+\left|R_{S}\right|\right)$, $\overline{R_{M}}=R_{M} /\left(\left|R_{L}\right|+\left|R_{M}\right|+\left|R_{S}\right|\right)$, and $\overline{R_{S}}=R_{S} /\left(\left|R_{L}\right|+\left|R_{M}\right|+\left|R_{S}\right|\right)$.

Midget cells were characterized as cone-opponent or nonopponent by comparing the sign of $\bar{R}_{L}$ and $\bar{R}_{M}$. ON and OFF midget cells were considered opponent when $\mathrm{L}$ - and $\mathrm{M}$-cone responses differed in sign (e.g., $-\bar{R}_{L},+\bar{R}_{M}$ or $+\bar{R}_{L},-\bar{R}_{m}$ ), and nonopponent when responses had the same sign (e.g., $+\bar{R}_{L}$, + $\bar{R}_{M}$ or $\left.-\bar{R}_{L},-\bar{R}_{M}\right)$. Consistent with our previous recordings in near-peripheral retina (Wool et al., 2018), our midget cell population was heterogeneously composed of both $\mathrm{L}-\mathrm{M}$ opponent (195 OFF, $44 \mathrm{ON}$ ) and nonopponent (61 OFF, $22 \mathrm{ON}$ ) cells (Fig. $7 A$ ). Comparing the relative unsigned proportion of S-, L-, and $\mathrm{M}$-cone inputs to each cell, we observed a distinct cluster of blue ON cells in which $S$-cone input comprised the majority of cone input $(0.70 \pm 0.19)$, against nonopponent $\mathrm{L}$ - and $\mathrm{M}$-cone inputs. By comparison, we found that midget cells formed a broad continuum of $\mathrm{L}-\mathrm{M}$ opponency, but also of S-cone sensitivity, par- 
A

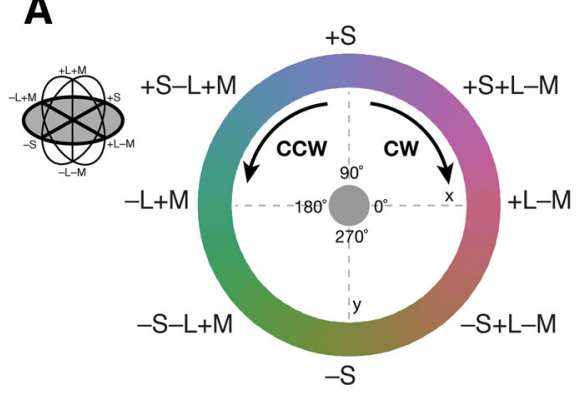

B
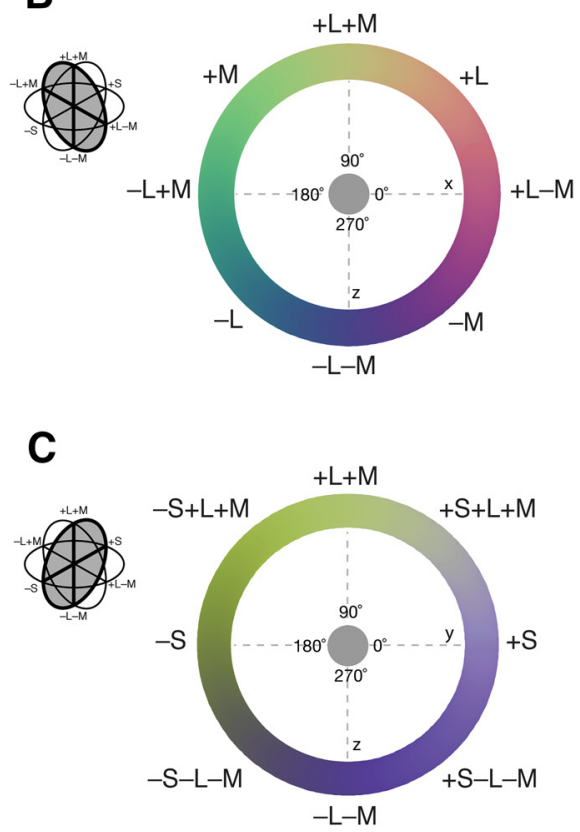

D

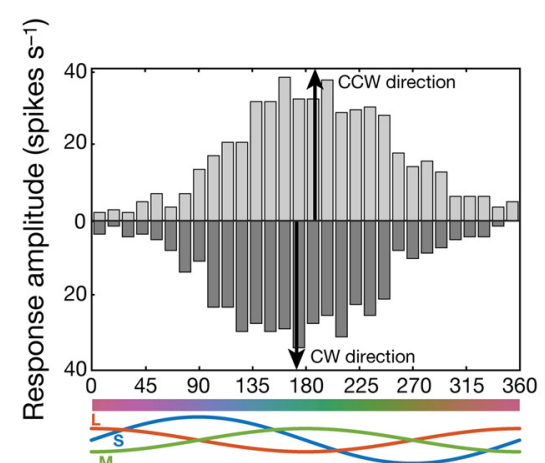

E

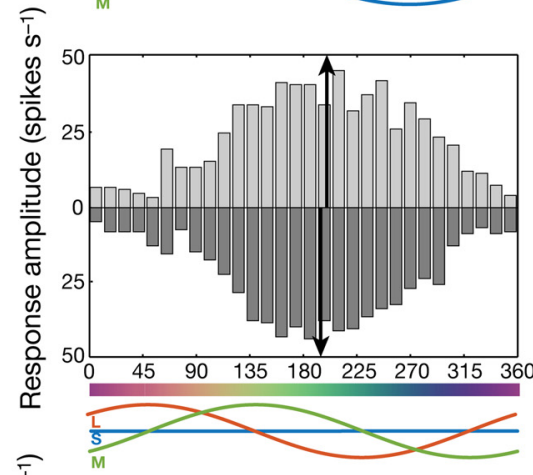

$F$

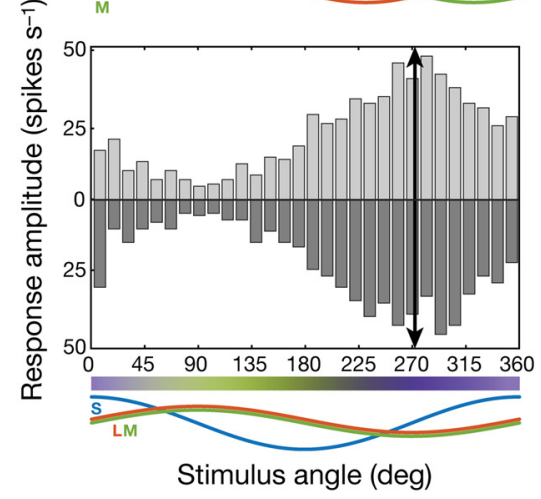

G

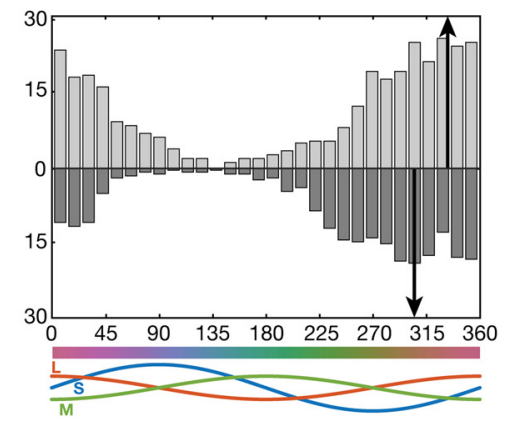

H
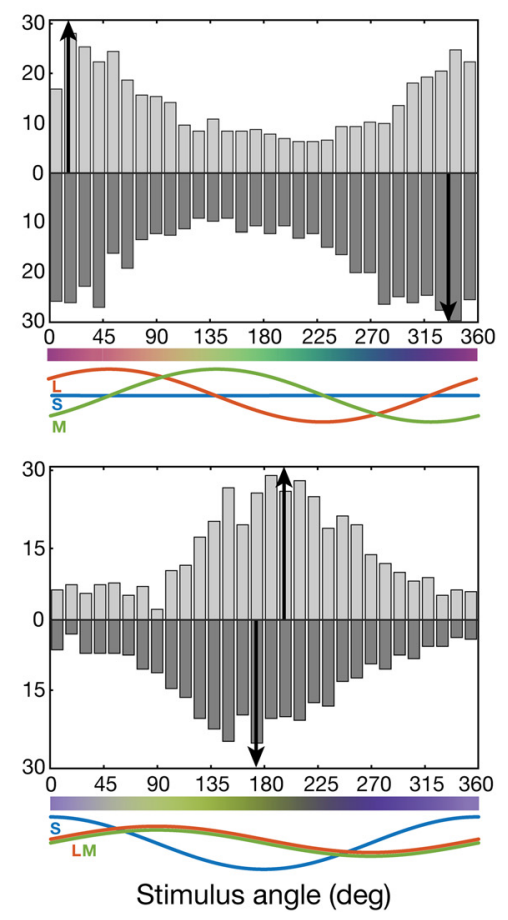

Figure 8. Measuring color preferences of individual ganglion cells. $\boldsymbol{A}-\boldsymbol{C}$, The chromaticity of a uniform, full-field stimulus was modulated in CW or CCW directions around circles in three intersecting planes of three-dimensional color space, $x y z$ : an $L-M$ versus $S$ isoluminant plane $(x y ; \boldsymbol{A})$, an $L-M$ versus $L+M$ plane $(y z ; \boldsymbol{B})$, and an $L+M$ versus $S$ plane $(x z ; \boldsymbol{C}$ ). $\boldsymbol{D}-\boldsymbol{F}$, Peristimulus time histograms reveal the color preference in each stimulus plane for an L cone-dominated, opponent OFF midget ganglion cell with absent S-cone input ( $61 \% \mathrm{~L}, 39 \% \mathrm{M}, 0 \% \mathrm{~S})$. The cell peaks near $180^{\circ}$ $(-\mathrm{L}+\mathrm{M})$ in the $x y$ plane $(\boldsymbol{D})$, near $135^{\circ}(+\mathrm{M}-\mathrm{L})$ in the $x z$ plane $(\boldsymbol{E})$, and near $270^{\circ}(-\mathrm{L}-\mathrm{M})$ in the $y z$ plane $(\boldsymbol{F})$. $\mathbf{G}-\boldsymbol{I}$, By contrast, peristimulus time histograms reveal the color preference in each stimulus plane for an $\mathrm{M}$ cone-dominated, opponent S-0FF midget ganglion cell with inputs from all three cones types $(23 \% \mathrm{~L}, 44 \% \mathrm{M}, 33 \% \mathrm{~S})$. This cell peaks near $315^{\circ}(-\mathrm{S},+\mathrm{L},-\mathrm{M})$ in the $x y$ plane $(\boldsymbol{G})$, near $0^{\circ}(+\mathrm{L},-\mathrm{M})$ in the $x z$ plane $(\boldsymbol{H})$, and near $180^{\circ}(-\mathrm{S})$ in the $y z$ plane $(\boldsymbol{I})$.

ticularly in OFF midget cells. The mean proportional S-cone input across ON midget cells was $0.04 \pm 0.05$, while across OFF midget cells the proportion was twice as high $(0.08 \pm 0.09)$. The stronger overall S-cone contribution to OFF midget receptive fields is visualized in Figure $7 \mathrm{~A}$ as a greater scatter of OFF midget cells into the orthogonal $\bar{R}_{S}$ axis; ON midget cells, by contrast, remain more closely clustered along the diagonals between the dominant $\mathrm{L}$ and $\mathrm{M}$ axes. To further quantify this tendency of OFF midget cells to exhibit stronger S-cone input than ON midget cells, we compared the cumulative distributions of $\mathrm{ON}$ and $\mathrm{OFF}$ midget cells as a function of unsigned proportional S-cone input, $\left|\bar{R}_{S}\right|$ (Fig. $7 B$ ). ON midget cells show a narrower distribution around weak or absent S-cone input, while S-cone input in OFF midget cells is more broadly distributed toward higher values of $\left|\bar{R}_{S}\right|$. This distribution of S-cone input between ON and OFF midget cells was statistically significantly different $(p=0.0013$, Kolmogorov-Smirnov test). Indeed, a much larger percentage of OFF midget cells showed proportional S-cone input of $>0.10$ :
$27.3 \%$ (70 of 256), compared with $10.6 \%$ (7 of 66) of ON midget cells.

Nonopponent S-OFF midget cells were much more prevalent in our sample compared with opponent S-OFF cells. OFF midget cells with proportional S-cone input of $>0.10$ comprised $45.9 \%$ (28 of 61 ) of all nonopponent OFF midget cells, but only $21.5 \%$ (42 of 195) of opponent OFF cells. Since the likelihood of encountering opponent or nonopponent midget cells is itself a function of eccentricity (Wool et al., 2018), we assessed whether this difference in detectable S-cone input between opponent and nonopponent OFF midget cells could also be attributed to a difference in retinal location. We found the mean eccentricity between these groups to be statistically indistinguishable (nonopponent cells: $33.3 \pm 6.6^{\circ}, n=24$; opponent cells: $30.7 \pm 6.8^{\circ}$, $n=34 ; p=0.15$, Student's $t$ test). While we did not further test whether nonopponent OFF midget cells have a greater likelihood of exhibiting S-cone input, the effect at least appears to be independent of eccentricity-dependent receptive-field sampling of 

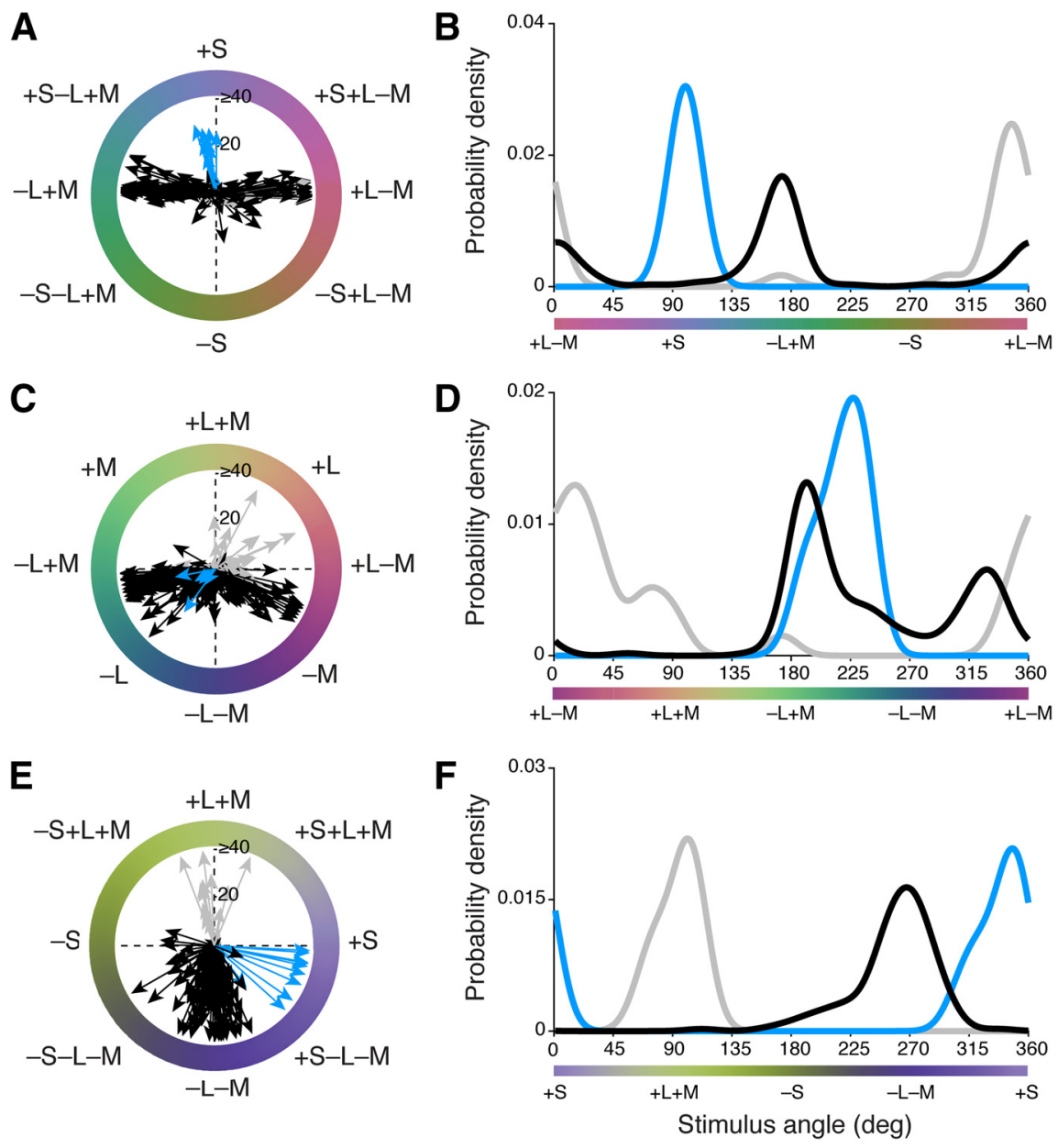

Figure 9. Distributions of color preferences across blue $0 \mathrm{~N}, 0 \mathrm{~N}$ midget, and OFF midget ganglion cells. $\boldsymbol{A}, \boldsymbol{B}$, Individual preferred vectors $(\boldsymbol{A})$ and kernel density estimation $(\boldsymbol{B})$ for cells in the xy plane. $\boldsymbol{C}, \boldsymbol{D}$, Individual preferred vectors $(\boldsymbol{C})$ and kernel density estimation $(\boldsymbol{D})$ for cells in the $x z$ plane. $\boldsymbol{E}, \boldsymbol{F}$, Individual preferred vectors $(\boldsymbol{E})$ and kernel density estimation $(\boldsymbol{F})$ for cells in the $y z$ plane. Blue, blue $0 \mathrm{~N}$ cells; gray, $0 \mathrm{~N}$ midget cells; black, OFF midget cells. Vector magnitude is expressed as firing rate (in spikes per second) and probability density is normalized for each cell type.

the cone mosaic. In any case, our analysis of cone-specific inputs to midget cells demonstrated that a substantial number of both opponent and nonopponent OFF midget cells exhibit not only classical L- and M-cone inputs, but in fact input from all three cone types. As the degree of L, M, and S sensitivity manifests heterogeneously across this class of $\mathrm{S}$ cone-sensitive midget ganglion cell, we sought to further probe these interactions by assigning a precise chromatic signature to each cell, identifying where these cells would fall in a complex, three-dimensional color space.

\section{S-OFF midget cells demonstrate complex, three-dimensional color tuning}

A major goal of this study was to determine how the three classical postreceptoral color mechanisms (as identified in LGN neurons; Derrington et al., 1984) combine at the level of the midget ganglion-cell receptive field to encode color sensitivity beyond $\mathrm{L}-\mathrm{M}$ (red-green) opponency. In a three-dimensional color space, $x y z$, defined by a cone-subtractive axis ( $\mathrm{L}-\mathrm{M}, x$-axis), an $\mathrm{S}$-cone isolating axis ( $y$-axis), and a cone-additive axis $(\mathrm{L}+\mathrm{M}$, $z$-axis), classical red-green opponent midget cells would simply project along the single $\mathrm{L}-\mathrm{M}$ dimension. While we observed many cells with such classical opponency, we also observed many midget ganglion cells with unbalanced $\mathrm{L}$ and $\mathrm{M}$ inputs, nonop- ponent $\mathrm{L}$ and $\mathrm{M}$ inputs, or even $\mathrm{S}$ inputs (Fig. 7A). We thus expected the color preferences of these cells to be heterogeneous and to project away from the single $\mathrm{L}-\mathrm{M}$ opponent axis.

To assess the strength of the three postreceptoral mechanisms in individual ganglion cells, and to visualize their color preferences in this three-dimensional color space, we used a set of slow-modulating sinusoidal stimuli (Sun et al., 2006) in which chromaticity was modulated around circles in three intersecting stimulus planes: an $(\mathrm{L}-\mathrm{M})$ versus $\mathrm{S}$ isoluminant plane ( $x y$; Fig. $8 A$ ), an $(\mathrm{L}-\mathrm{M})$ versus $(\mathrm{L}+\mathrm{M})$ plane $(x z$; Fig. $8 B)$, and an $(\mathrm{L}+\mathrm{M})$ versus $S$ plane $(y z$; Fig. $8 C)$. Each stimulus was presented as a uniform field (800-1200 $\mu \mathrm{m}$ ) encompassing the receptive-field center and surround of the cell. For example, in a classical OFF midget cell with balanced and opponent $\mathrm{L}$ and $\mathrm{M}$ inputs and no $\mathrm{S}$ input, PSTHs show the preference of the cell for $-\mathrm{L}+\mathrm{M}$ stimuli in each of the three planes, as follows: near $180^{\circ}(-\mathrm{L}+\mathrm{M})$ in the $x y$ plane (Fig. $8 D)$, near $135^{\circ}(+\mathrm{M}-\mathrm{L})$ in the $x z$ plane (Fig. $8 E$ ), and near $270^{\circ}(-\mathrm{L}-\mathrm{M})$ in the $y z$ plane (Fig. $8 F$ ). By contrast, an example opponent OFF midget cell with S-cone input shows significantly shifted preferred vectors that reflect not only the preference of the cell for opponent $-\mathrm{M}+\mathrm{L}$ stimuli, but also additional S-cone sensitivity to its receptive field, as follows: near $315^{\circ}$ $(-\mathrm{S},+\mathrm{L},-\mathrm{M})$ in the $x y$ plane (Fig. $8 G)$, near $0^{\circ}(+\mathrm{L},-\mathrm{M})$ in the $x z$ plane (Fig. $8 H)$, and near $180^{\circ}(-\mathrm{S})$ in the $y z$ plane (Fig. 8I).

We recorded spike activity from 223 retinal ganglion cells ( 10 blue $\mathrm{ON}$ cells, 191 OFF midget cells, 22 ON midget cells) during presentation of slow-modulating stimuli in the three stimulus planes. Initially, we observed that the color preferences of OFF midget, ON midget, and blue ON ganglion cells were largely heterogeneous both within and across cell type, even in response to the same color stimulus (Fig. 9). Some clustering of preferred vectors was evident in each stimulus plane, as follows: in the $\mathrm{L}-\mathrm{M}$ versus $\mathrm{S}$ isoluminant $(x y)$ plane (Fig. $9 A, B)$, blue ON cells $(n=9)$ cluster closely to $90^{\circ}(+\mathrm{S})$, while both ON $(n=19)$ and OFF midget cells $(n=183)$ heavily cluster at the two cone-opponent directions near $0^{\circ}(+\mathrm{L}-\mathrm{M})$ and $180^{\circ}(-\mathrm{L}+\mathrm{M})$. Notably, a small number of OFF cells show that variable preferred vectors depart from the cone-opponent axis toward $270^{\circ}(-\mathrm{S})$, with a color signature opposite to blue ON cells (Fig. 9A). While kernel density estimation successfully identified the bimodal distribution of $\mathrm{ON}$ and OFF midget cells (OFF: $172^{\circ}$ and $2^{\circ} ; \mathrm{ON}: 172^{\circ}$ and $347^{\circ}$ ) as well as the unimodal distribution of blue $\mathrm{ON}$ cells $\left(101^{\circ}\right)$, the low density of OFF midget cells in the intermediate directions toward $-\mathrm{S}$ $\left(225-315^{\circ}\right)$ underlines the overall scarcity of S-OFF cells in our population (Fig. 9B).

In the $\mathrm{L}-\mathrm{M}$ versus $\mathrm{L}+\mathrm{M}(x z)$ plane (Fig. $9 C, D)$, blue $\mathrm{ON}$ cells $(n=5)$ cluster at $225^{\circ}$, where the $(-\mathrm{L}-\mathrm{M})$ component of these cells is maximally stimulated. ON $(n=22)$ and OFF $(n=186)$ 


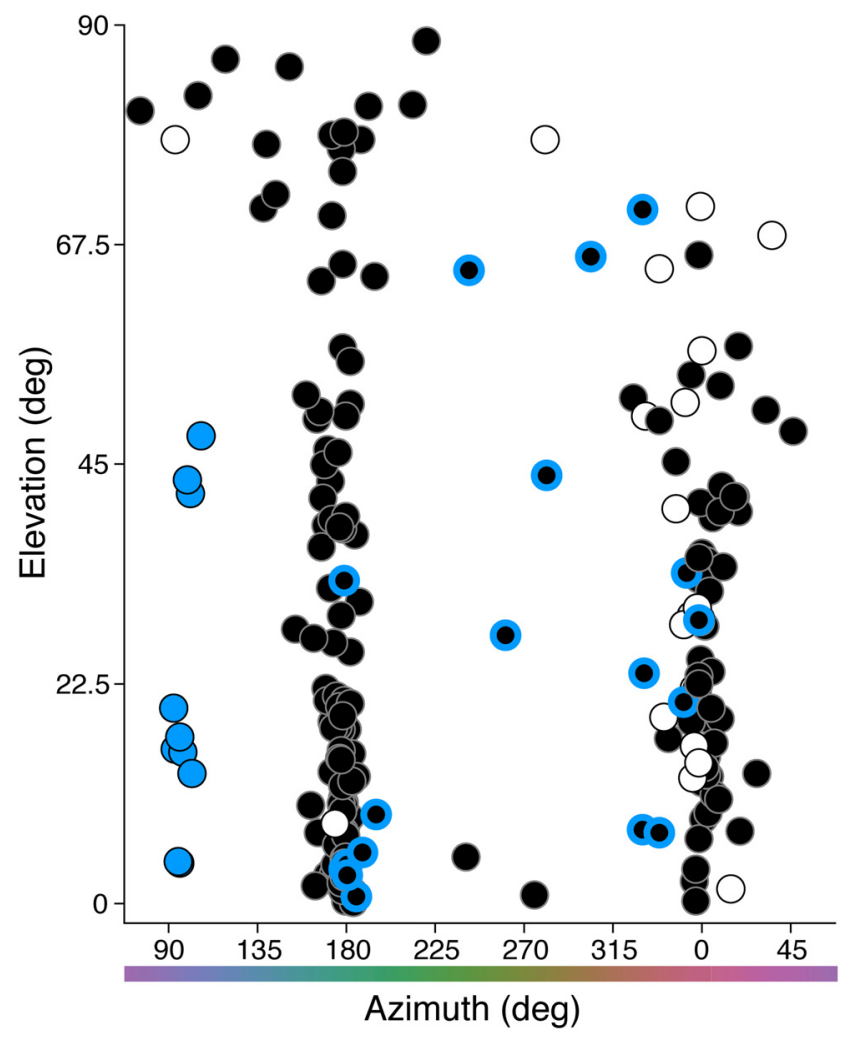

Figure 10. Three-dimensional color signatures of blue $\mathrm{ON}, \mathrm{ON}$ midget, and $0 \mathrm{FF}$ midget cells. The preferred azimuth and elevation of each cell was computed from its preferred $[x, y, z]$ vector in Cartesian coordinates, as generated from its preferred vectors across the three individual stimulus planes (see Fig. 9). Blue circles, blue $\mathrm{ON}$ cells; white circles, $\mathrm{ON}$ midget cells; black circles, OFF midget cells. Black/blue circles denote $0 \mathrm{FF}$ midget cells initially identified as $S$ cone sensitive in Figure 1.

midget cells show a very broad distribution of preferred vectors that span both cone-opponent $\left(0^{\circ}, 180^{\circ}\right)$ and nonopponent $\left(90^{\circ}\right.$, $270^{\circ}$ ) directions, illustrating the heterogeneity of opponent responses in midget ganglion cells (Solomon et al., 2005; Buzás et al., 2006; Martin et al., 2011; Wool et al., 2018). The estimated preferred-vector kernels clearly identified a sharp unimodal distribution of blue $\mathrm{ON}$ cells $\left(228^{\circ}\right)$, and a multimodal distribution of ON and OFF midget cells, as follows: modes at two L-dominated cone-opponent directions (OFF: $194^{\circ}$ and $327^{\circ}$; $\mathrm{ON}: 174^{\circ}$ and $\left.15^{\circ}\right)$, as well as a nonzero density for intermediate, nonopponent L-dominated $+\mathrm{L}+\mathrm{M}\left(45-90^{\circ}\right)$ and $-\mathrm{L}-\mathrm{M}(225-$ $270^{\circ}$ ) directions.

In the $\mathrm{L}+\mathrm{M}$ versus $\mathrm{S}(y z)$ plane (Fig. $9 E, F)$, blue $\mathrm{ON}$ cells $(n=$ 10) again show a single cluster near $315^{\circ}$, where opponent $+S$ and $-\mathrm{L}-\mathrm{M}$ mechanisms are maximally stimulated. ON midget cells $(n=13)$ narrowly cluster at $90^{\circ}(+\mathrm{L}+\mathrm{M})$; by contrast, OFF midget cells $(n=143)$ show a much broader distribution of preferred vectors that span both $-\mathrm{L}-\mathrm{M}$ and $-\mathrm{S}$ mechanisms (Fig. 9E). Compared with the bimodal distributions observed in other stimulus planes, preferred-vector kernels for ON midget, OFF midget, and blue ON cells are unimodal in the $x z$ plane, but with varying broadness of their distribution. The preferredvector kernels for blue $\mathrm{ON}$ cells $\left(348^{\circ}\right)$ and $\mathrm{ON}$ midget cells $\left(101^{\circ}\right)$ are relatively narrow and normally distributed; by contrast, while the kernel for OFF midget cells shows clear mode at $268^{\circ}$, there is an obvious leftward skew of the distribution, reflecting the substantial number of S-OFF cells that exhibit tuning toward the $-\mathrm{S}$ direction $\left(180^{\circ}\right.$; Fig. $\left.9 F\right)$.
After computing the preferred vector of each cell to each stimulus (Fig. 9), we computed the vector projection of each cell in three-dimensional color space (Eqs. 7-9 and Materials and Methods) and reported this in the following spherical coordinates: azimuth $\left(\varphi\right.$; range on the $x y$ plane, $0-360^{\circ}$ ) and absolute elevation $\left(\theta\right.$; range, $0-90^{\circ}$, where $0^{\circ}$ lies on the $x y$ plane and $90^{\circ}$ lies on the $z$-axis). Figure 10 shows the distribution of preferred directions of all ganglion cells in three-dimensional color space.

The majority of cells fall in three clusters along the azimuth, as follows: blue ON cells fall at $\varphi=90^{\circ}(+S)$, while ON and OFF midget ganglion cells fall in columns at $\varphi=0^{\circ}(+\mathrm{L}-\mathrm{M})$ and $\varphi=$ $180^{\circ}(-\mathrm{L}+\mathrm{M})$. Moreover, midget cells also show variable tradeoff between being strongly $\mathrm{L}-\mathrm{M}$ cone-opponent $\left(\theta=0^{\circ}\right)$ and nonopponent $\left(\theta=90^{\circ}\right)$, which is consistent with previous studies (Solomon et al., 2005; Buzás et al., 2006; Field et al., 2010; Martin et al., 2011; Wool et al., 2018). In contrast to the narrow clusters of cells at azimuths of $0^{\circ}, 90^{\circ}$, and $180^{\circ}$, a sparse population of OFF midget cells are widely scattered between $0^{\circ}$ and $180^{\circ}$, indicating a degree of S-OFF input that varies considerably to the receptive fields of these OFF midget cells. These cells also range from strongly chromatic $\left(\mathrm{L}-\mathrm{M}, \theta=0^{\circ}\right)$ to strongly achromatic $\left(\mathrm{L}+\mathrm{M}, \theta=90^{\circ}\right)$; this is consistent with our earlier finding that $\mathrm{S}$-cone inputs are observed across both opponent and nonopponent OFF midget cells.

\section{Discussion}

By contrast with the S-ON pathway in primate retina, where S cone-selective blue cone bipolar cells synapse with the small bistratified ganglion cell (Dacey and Lee, 1994; Calkins et al., 1998), a comparable S-OFF pathway does not appear to lie within an anatomically discrete cell class. Instead, our anatomical and physiological results confirm that in the macaque monkey retina, S-OFF signals are propagated by a subpopulation of OFF midget ganglion cells with connectivity to $S$ cones (Calkins, 2001; Klug et al., 2003; Field et al., 2010; Dacey et al., 2014; Tsukamoto and Omi, 2015).

In a previous EM study, Klug et al. (2003) identified five S cones by their connectivity to blue cone bipolar cells and then showed that each of these cones contacted an OFF midget bipolar cell; two of these cells were completely reconstructed and indistinguishable from OFF midget bipolar cells connected to L/M cones. Thus, the origin of a major S-OFF visual pathway in the primate retina appeared to be identified. However, subsequent work in marmoset retina-where $S$ cones were identified by S-opsin expression - showed no such connection to OFF midget bipolar cells (Lee et al., 2005) despite a homologous S-ON small bistratified pathway in this primate species (Ghosh et al., 1997). These results, along with an earlier result suggesting a similar absence of an OFF midget bipolar at presumed S-cone pedicles in the human retina (Kolb et al., 1997) as well as recordings from LGN in both macaque and marmoset identifying a non-midget S-OFF pathway (Szmajda et al., 2006; Tailby et al., 2008a,b) raised new questions about the retinal substrate for the S-OFF signal in color vision (Lee et al., 2010; Neitz and Neitz, 2011). Later EM reconstructions and physiological data from the retinal periphery (Field et al., 2010; Tsukamoto and Omi, 2015) challenged this apparent absence by again highlighting a sparse S-cone input to the OFF midget circuit.

We took advantage of recent findings that S-cone pedicles uniquely lack the short telodendria that enable $\mathrm{L}$ and $\mathrm{M}$ cones to interconnect via gap junctions (O'Brien et al., 2012) to unequivocally identify S-cone pedicles and to create a larger dataset of S-cone circuits that clearly confirm and extend the results of the 


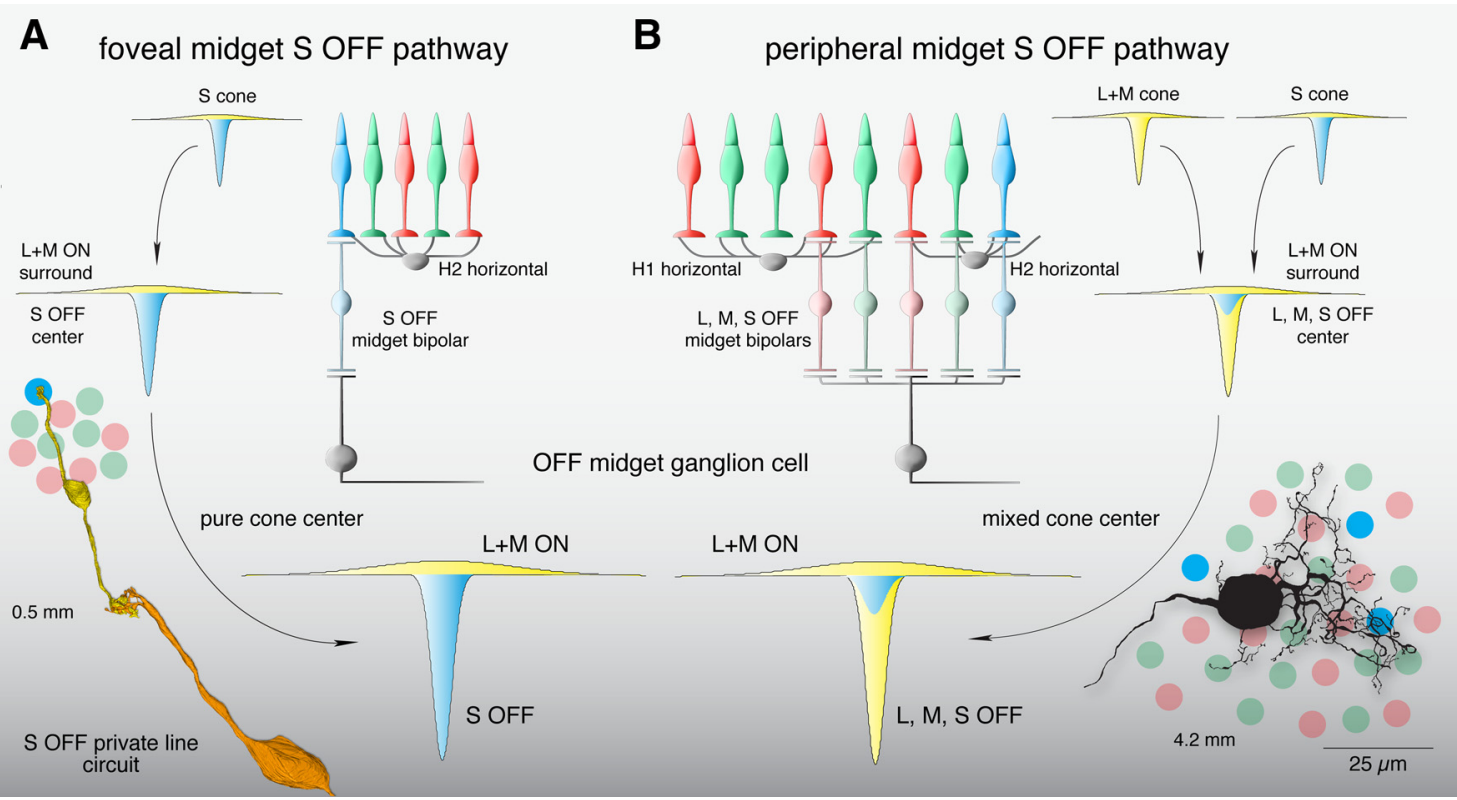

Figure 11. Summary of the OFF midget circuit illustrating a synaptic basis for S-OFF opponent pathways in foveal and near-peripheral retinae. $A, A n L+M O N$ surround in the $S$ cone is formed by $\mathrm{H} 2$ horizontal cell negative feedback to $\mathrm{S}$ cones (Packer et al., 2010). This receptive field structure is transmitted via an S-OFF midget bipolar cell and, subsequently, an OFF midget ganglion cell, creating an S-OFF private line circuit (curved arrows). These OFF midget ganglion cells would thus show center-surround organization and S OFF, L+M ON opponency. Inset at lower left shows a schematic foveal cone mosaic of $S$ (blue), $L($ red), and $M$ (green) cones in relation to an S-0FF midget circuit reconstruction taken from Figure $3, B$ and C. $B$, In the retinal periphery, $L+M$ ON surrounds are formed in each cone via negative feedback from $\mathrm{H} 1$ horizontal cells (to $\mathrm{L}$ and $\mathrm{M}$ cones) and $\mathrm{H} 2$ horizontal cells (to $S$ cones), which are inherited by individual midget bipolar cells and transmitted to midget ganglion cells (curved arrows), which collect input from three to five midget bipolar cells in the near retinal periphery ( $4 \mathrm{~mm}$ from the fovea; Wool et al., 2018). Converging S, L, and $\mathrm{M}$ cone inputs create a receptive field in the midget ganglion cell with complex chromatic properties- $(S+L)$ OFF, $M$ ON; $(S+M)$ OFF, $L$ ON; or $(S+L+M)$ OFF- depending on the relative strength of individual cone inputs (Wool et al., 2018). Inset at lower right shows the peripheral cone mosaic, as in $A$, in spatial relation to a near peripheral midget ganglion-cell dendritic tree at $\sim 4 \mathrm{~mm}$ from the foveal center.

study by Klug et al. (2003) (Fig. 11A). Moreover, we found fewer ribbon synapses at both the $\mathrm{S}$-cone pedicle and the associated OFF midget bipolar axon terminal compared with neighboring $\mathrm{L} / \mathrm{M}$ circuits. The S-cone pathway seems not only distinct in its postsynaptic circuit, but also at the quantitative level of ribbon synapse deployment. Given the negative evidence from both human and marmoset, our results suggest significant differences in retinal circuitry across primate groups. It will be critical to use volume EM methods to determine the S-cone connectivity in both marmosets and, most importantly, in humans, where connectomics is now being used to characterize foveal cell types and circuits (Dacey et al., 2017; Packer et al., 2017).

If private-line S-OFF midget circuits were present in the human retina, it would suggest spatial resolution limited by the S-cone sampling array for this pathway. Peak foveal acuity mediated by S-cones is $\sim 10$ cpd (Stromeyer et al., 1978; Hess et al., 1989; Metha and Lennie, 2001), exceeding the resolution afforded by S-cone spacing. However acuity drops well below this limit by $2-3^{\circ}$ eccentricity (where private-line midget circuitry is present), and this reduction is more consistent with the extensive postreceptoral pooling of the blue ON ganglion cell (Metha and Lennie, 2001), placing it in agreement with other studies of S cone-mediated acuity (Anderson et al., 2002; Beirne et al., 2005). Other measurements suggest that beyond $5^{\circ}$, the S-OFF pathway actually shows lower resolution than its S-ON counterpart (Vassilev et al., 2003; Zlatkova et al., 2008), which is consistent with the rapid postreceptoral pooling of midget ganglion cells that supplants private-line connectivity at greater eccentricities.

In the temporal domain, differences between S-ON and S-OFF signaling do point to their propagation by parallel pathways that sum S cone inputs differently. While some studies ob- served faster responses to S-OFF stimuli than to S-ON stimuli (McKeefry et al., 2003; Wool et al., 2015), others have reported slower, weaker S-OFF responses both psychophysically (Shinomori and Werner, 2008) and in human electroretinography (Maguire et al., 2018). Such a small and sluggish S-OFF response may be due to either the reduction or absence of this pathway in human retina or to greater variability in the connection strength of the $\mathrm{S}$ cones to the OFF midget pathway with increasing eccentricity.

In the macaque retina, S-OFF midget cells in the central retina form a private-line pathway, but in the periphery the midget cell dendritic tree enlarges and thus draws converging input from L, $\mathrm{M}$, and S cone-connecting midget bipolar cells (Jusuf et al., 2006; Tsukamoto and Omi, 2015; Fig. 11B). Consistent with this picture, we found that the midget $\mathrm{S}$-cone signal was variable in strength but invariant in phase, thus, the OFF sign of S-cone sensitivity across OFF-center midget cells is consistent with an S-cone contribution to OFF midget ganglion cells via midget bipolar inputs to the excitatory receptive-field center. The spatial extent of S-cone input also supports this interpretation, as the cutoff frequency of spatial tuning curves for S-cone gratings closely matches that of tuning curves to $\mathrm{L}+\mathrm{M}$ gratings in peripheral OFF midgets. This suggests that in the near periphery where midget dendritic fields receive convergent input from multiple midget bipolar cells, $\mathrm{L}, \mathrm{M}$, and $\mathrm{S}$ cones all contribute to the S-OFF midget ganglion-cell receptive field center (Field et al., 2010).

The variable input strength from $S$ cones to OFF midget ganglion cells is consistent with the conclusion that the midget circuit samples nonselectively form the cone mosaic (Calkins and Sterling, 1999; Crook et al., 2011; Wool et al., 2018). Circuit reconstructions in peripheral retina show that S-cone input is variable 
across neighboring OFF midget ganglion cells, in a manner seemingly dependent on the underlying cone mosaic (Tsukamoto and Omi, 2015). If we assume an S-cone sampling mechanism akin to that for $\mathrm{L}$ and $\mathrm{M}$ cones, and limited by the sparse S-cone mosaic (Curcio et al., 1991), we would predict that for midget cells in the far periphery, the likelihood of any S-cone contact would increase, but that the relative strength of S-cone input would decline as overall cone inputs (the great majority $\mathrm{L}$ and $\mathrm{M}$ ) increase. On the other hand, we would predict this tradeoff to be reversed foveally, where a private-line OFF midget circuit is present for every $\mathrm{S}$ cone, but at a rate in keeping with the low density of $\mathrm{S}$ cones. Given that $\mathrm{S}$ cones already display an L/M antagonistic surround via negative feedback from horizontal cells (Packer et al., 2010, Fig. 11A), a sparse population of foveal S-OFF center, $\mathrm{L}+\mathrm{M}$ ON surround midget ganglion cells would provide color tuning directly opposing the spatially coextensive S-ON, L+MOFF fields of blue ON cells.

We found that the S-OFF connection broadly influences the color-sensitive properties of individual OFF midget cells, giving rise to a population with strongly heterogeneous color preferences that do not lie along the cardinal S versus $\mathrm{L}+\mathrm{M}$ and $\mathrm{L}$ versus M chromatic axes (Krauskopf et al., 1982; Derrington et al., 1984). Compared with the consistent $S$ versus $L+M$ tuning we observed in blue ON cells, S-OFF midget cells showed L+S OFF, M ON tuning ( $\mathrm{L}$ and $\mathrm{S}$ cones in phase), $\mathrm{M}+\mathrm{S}$ OFF, $\mathrm{L}$ ON tuning ( $\mathrm{M}$ and $\mathrm{S}$ in phase), or $\mathrm{L}+\mathrm{M}+\mathrm{S}$ OFF tuning (all cone types in phase). The heterogeneity of phase relationships observed across $\mathrm{L}, \mathrm{M}$, and S cone mechanisms in S-OFF midget cells may help to explain why psychophysical studies find that S-cone input can be synergistic with either L-cone input (Stromeyer et al., 1998; Goddard et al., 2010; Danilova and Mollon, 2012) or M-cone input (Conway, 2001; Solomon and Lennie, 2005; Horwitz et al., 2007; Lafer-Sousa et al., 2012). These studies measured response amplitudes to modulating ON-OFF gratings assuming symmetry between the poles of various color axes, making it difficult to determine the individual contributions of putative $\mathrm{ON}$ and $\mathrm{OFF}$ mechanisms to color sensitivity. Our measurements of color preference reveal that such poles are rarely symmetric and often driven by multiple cell subtypes (Fig. 9). In any case, our findings suggest that any reported "tilt" off the cardinal $\mathrm{L}-\mathrm{M}$ axis might be accounted for by the mixed color properties of S-OFF midget cells (Fig. 10) or their LGN relay cell counterparts (Tailby et al., 2008a).

While the OFF midget circuit may be the dominant pathway for S-OFF signals and set the limit on S-cone spatial resolution, other pathways may also contribute. Melanopsin-expressing intrinsically photosensitive ganglion cells show an S-OFF input (Dacey et al., 2005), and some nonmidget diffuse bipolar types also sparsely contact $\mathrm{S}$ cones in addition to $\mathrm{L}$ and $\mathrm{M}$ cones (Calkins and Sterling, 2007; Lee and Grünert, 2007). This suggests additional S-OFF signals involved in color processing may be transmitted to the LGN by ganglion-cell types that remain to be characterized (Dacey et al., 2003).

\section{References}

Anderson RS, Zlatkova MB, Beirne RO (2002) The contrast sensitivity function for detection and resolution of blue-on-yellow gratings in foveal and peripheral vision. Ophthalmic Physiol Opt 22:420-426.

Banks MS, Sekuler AB, Anderson SJ (1991) Peripheral spatial vision: limits imposed by optics, photoreceptors, and receptor pooling. J Opt Soc Am A 8:1775-1787.

Baylor DA, Lamb TD, Yau KW (1979) Responses of retinal rods to single photons. J Physiol 288:613-634.
Baylor DA, Nunn BJ, Schnapf JL (1987) Spectral sensitivity of cones of the monkey macaca fascicularis. J Physiol 390:145-160.

Beirne RO, Zlatkova MB, Anderson RS (2005) Changes in human shortwavelength-sensitive and achromatic resolution acuity with retinal eccentricity and meridian. Vis Neurosci 22:79-86.

Berens P (2009) CircStat: a MATLAB toolbox for circular statistics. J Stat Softw 31:1-21.

Boycott BB, Wässle H (1991) Morphological classification of bipolar cells of the primate retina. Eur J Neurosci 3:1069-1088.

Brainard D, Stockman A (2010) Colorimetry. In: The Optical Society of America Handbook of Optics, Ed 3 (Bass M, DeCusatis C, Enoch J, Lakshminarayanan V, Li G, Macdonald C, Mahajan V, van Stryland E, eds), pp 10.1-10.56. New York: McGraw Hill.

Bumsted K, Hendrickson A (1999) Distribution and development of shortwavelength cones differ between Macaca monkey and human fovea. J Comp Neurol 403:502-516.

Buzás P, Blessing EM, Szmajda BA, Martin PR (2006) Specificity of M and L cone inputs to receptive fields in the parvocellular pathway: random wiring with functional bias. J Neurosci 26:11148-11161.

Calkins DJ (2001) Seeing with S cones. Prog Retin Eye Res 20:255-287.

Calkins DJ, Sterling P (1999) Evidence that circuits for spatial and color vision segregate at the first retinal synapse. Neuron 24:313-321.

Calkins DJ, Sterling P (2007) Microcircuitry for two types of achromatic ganglion cell in primate fovea. J Neurosci 27:2646-2653.

Calkins DJ, Schein SJ, Tsukamoto Y, Sterling P (1994) M and L cones in macaque fovea connect to midget ganglion cells by different numbers of excitatory synapses. Nature 371:70-72.

Calkins DJ, Tsukamoto Y, Sterling P (1998) Microcircuitry and mosaic of a blue-yellow ganglion cell in the primate retina. J Neurosci 18:3373-3385.

Cardona A, Saalfeld S, Schindelin J, Arganda-Carreras I, Preibisch S, Longair M, Tomancak P, Hartenstein V, Douglas RJ (2012) TrakEM2 software for neural circuit reconstruction. PLoS One 7:e38011.

Chen S, Li W (2012) A color-coding amacrine cell may provide a blue-off signal in a mammalian retina. Nat Neurosci 15:954-956.

Conway BR (2001) Spatial structure of cone inputs to color cells in alert macaque primary visual cortex (V-1). J Neurosci 21:2768-2783.

Crook JD, Peterson BB, Packer OS, Robinson FR, Gamlin PD, Troy JB, Dacey DM (2008) The smooth monostratified ganglion cell: evidence for spatial diversity in the Y-cell pathway to the lateral geniculate nucleus and superior colliculus in the macaque monkey. J Neurosci 28:12654-12671.

Crook JD, Davenport CM, Peterson BB, Packer OS, Detwiler PB, Dacey DM (2009) Parallel ON and OFF cone bipolar inputs establish spatially coextensive receptive field structure of blue-yellow ganglion cells in primate retina. J Neurosci 29:8372-8387.

Crook JD, Manookin MB, Packer OS, Dacey DM (2011) Horizontal cell feedback without cone type-selective inhibition mediates "red-green" color opponency in midget ganglion cells of the primate retina. J Neurosci 31:1762-1772.

Curcio CA, Allen KA, Sloan KR, Lerea CL, Hurley JB, Klock IB, Milam AH (1991) Distribution and morphology of human cone photoreceptors stained with anti-blue opsin. J Comp Neurol 312:610-624.

Dacey DM (1993) The mosaic of midget ganglion cells in the human retina. J Neurosci 13:5334-5355.

Dacey DM, Lee BB (1994) The "blue-on" opponent pathway in primate retina originates from a distinct bistratified ganglion cell type. Nature 367:731-735.

Dacey DM, Petersen MR (1992) Dendritic field size and morphology of midget and parasol ganglion cells of the human retina. Proc Natl Acad Sci U S A 89:9666-9670.

Dacey DM, Peterson BB, Robinson FR, Gamlin PD (2003) Fireworks in the primate retina: in vitro photodynamics reveals diverse LGN-projecting ganglion cell types. Neuron 37:15-27.

Dacey DM, Liao HW, Peterson BB, Robinson FR, Smith VC, Pokorny J, Yau KW, Gamlin PD (2005) Melanopsin-expressing ganglion cells in primate retina signal colour and irradiance and project to the LGN. Nature 433:749-754.

Dacey DM, Crook JD, Packer OS (2014) Distinct synaptic mechanisms create parallel S-ON and S-OFF color opponent pathways in the primate retina. Vis Neurosci 31:139-151.

Dacey DM, Packer OS, Schalek R, Curcio CA, Wong RO, Dowling JE, Lichtman JW (2017) Connectomic reconstruction links human foveal cones 
to distinct circuitry in the center of the foveal pit. Invest Ophthalmol Vis Sci 58:1036.

Dacey D, Packer OS, Diller L, Brainard D, Peterson B, Lee B (2000) Center surround receptive field structure of cone bipolar cells in primate retina. Vision Res 40:1801-1811.

Danilova MV, Mollon JD (2012) Cardinal axes are not independent in color discrimination. J Opt Soc Am A 29:A157.

Dartnall H (1972) Photosensitivity. In: Photochemistry of Vision (Dartnall H, ed), pp 122-145. New York: Springer.

Derrington AM, Krauskopf J, Lennie P (1984) Chromatic mechanisms in lateral geniculate nucleus of macaque. J Physiol 357:241-265.

Diller L, Packer OS, Verweij J, McMahon MJ, Williams DR, Dacey DM (2004) $\mathrm{L}$ and $\mathrm{M}$ cone contributions to the midget and parasol ganglion cell receptive fields of macaque monkey retina. J Neurosci 24:1079-1088.

Enroth-Cugell C, Robson JG, Schweitzer-Tong DE, Watson AB (1983) Spatio-temporal interactions in cat retinal ganglion cells showing linear spatial summation. J Physiol 341:279-307.

Field GD, Gauthier JL, Sher A, Greschner M, Machado TA, Jepson LH, Shlens J, Gunning DE, Mathieson K, Dabrowski W, Paninski L, Litke AM, Chichilnisky EJ (2010) Functional connectivity in the retina at the resolution of photoreceptors. Nature 467:673-677.

Ghosh KK, Martin PR, Grünert U (1997) Morphological analysis of the blue cone pathway in the retina of a new world monkey, the marmoset Callithrix jacchus. J Comp Neurol 379:211-225.

Goddard E, Mannion DJ, McDonald JS, Solomon SG, Clifford CW (2010) Combination of subcortical color channels in human visual cortex. J Vis 10(5):25, 1-17.

Herr S, Klug K, Sterling P, Schein S (2003) Inner S-cone bipolar cells provide all of the central elements for $\mathrm{S}$ cones in macaque retina. J Comp Neurol 457:185-201.

Hess RF, Mullen KT, Zrenner E (1989) Human photopic vision with only short wavelength cones: post-receptoral properties. J Physiol 417:151172.

Horwitz GD, Chichilnisky EJ, Albright TD (2007) Cone inputs to simple and complex cells in V1 of awake macaque. J Neurophysiol 97:30703081.

Jusuf PR, Martin PR, Grünert U (2006) Synaptic connectivity in the midgetparvocellular pathway of primate central retina. J Comp Neurol 494: $260-274$.

Klug K, Herr S, Ngo IT, Sterling P, Schein S (2003) Macaque retina contains an S-cone OFF midget pathway. J Neurosci 23:9881-9887.

Kolb H, Dekorver L (1991) Midget ganglion cells of the parafovea of the human retina: a study by electron microscopy and serial section reconstructions. J Comp Neurol 303:617-636.

Kolb H, Goede P, Roberts S, McDermott R, Gouras P (1997) Uniqueness of the $\mathrm{S}$-cone pedicle in the human retina and consequences for color processing. J Comp Neurol 386:443-460.

Kouyama N, Marshak DW (1992) Bipolar cells specific for blue cones in the macaque retina. J Neurosci 12:1233-1252.

Krauskopf J, Williams DR, Heeley DW (1982) Cardinal directions of color space. Vision Res 22:1123-1131.

Lafer-Sousa R, Liu YO, Lafer-Sousa L, Wiest MC, Conway BR (2012) Color tuning in alert macaque $\mathrm{V} 1$ assessed with $\mathrm{AMRI}$ and single-unit recording shows a bias toward daylight colors. J Opt Soc Am A 29:657-670.

Lee BB, Martin PR, Grünert U (2010) Retinal connectivity and primate vision. Prog Retin Eye Res 29:622-639.

Lee S, Grünert U (2007) Connections of diffuse bipolar cells in primate retina are biased against S-cones. J Comp Neurol 502:126-140.

Lee SC, Telkes I, Grünert U (2005) S-cones do not contribute to the OFFmidget pathway in the retina of the marmoset, Callithrix jacchus. Eur J Neurosci 22:437-447.

Maguire J, Parry NRA, Kremers J, Murray IJ, McKeefry D (2018) Human S-cone electroretinograms obtained by silent substitution stimulation. J Opt Soc Am A 35:B11-B18.

Mariani AP (1984) Bipolar cells in monkey retina selective for the cones likely to be blue-sensitive. Nature 308:184-186.

Martin PR, Blessing EM, Buzás P, Szmajda BA, Forte JD (2011) Transmission of colour and acuity signals by parvocellular cells in marmoset monkeys. J Physiol 589:2795-2812.
McKeefry DJ, Parry NR, Murray IJ (2003) Simple reaction times in color space: the influence of chromaticity, contrast, and cone opponency. Invest Ophthalmol Vis Sci 44:2267-2276.

McMahon MJ, Packer OS, Dacey DM (2004) The classical receptive field surround of primate parasol ganglion cells is mediated primarily by a non-GABAergic pathway. J Neurosci 24:3736-3745.

Metha AB, Lennie P (2001) Transmission of spatial information in S-cone pathways. Vis Neurosci 18:961-972.

Neitz J, Neitz M (2011) The genetics of normal and defective color vision. Vision Res 51:633-651.

O'Brien JJ, Chen X, Macleish PR, O'Brien J, Massey SC (2012) Photoreceptor coupling mediated by connexin 36 in the primate retina. J Neurosci 32:4675-4687.

Packer OS, Verweij J, Li PH, Schnapf JL, Dacey DM (2010) Blue-yellow opponency in primate $S$ cone photoreceptors. J Neurosci 30:568-572.

Packer OS, Schalek R, Wong RO, Curcio CA, Dowling JE, Lichtman JW, Dacey DM (2017) Restricted cone connections of horizontal cells in the human foveal center. Invest Ophthalmol Vis Sci 58:1035.

Schanda J (2007) Colorimetry: understanding the CIE system. Hoboken, NJ: Wiley.

Shapiro MB, Schein SJ, de Monasterio FM (1985) Regularity and structure of the spatial pattern of blue cones of macaque retina. J Am Stat Assoc 80:803-814.

Sher A, DeVries SH (2012) A non-canonical pathway for mammalian bluegreen color vision. Nat Neurosci 15:952-953.

Shinomori K, Werner JS (2008) The impulse response of S-cone pathways in detection of increments and decrements. Vis Neurosci 25:341-347.

Solomon SG, Lennie P (2005) Chromatic gain controls in visual cortical neurons. J Neurosci 25:4779-4792.

Solomon SG, Lee BB, White AJ, Rüttiger L, Martin PR (2005) Chromatic organization of ganglion cell receptive fields in the peripheral retina. J Neurosci 25:4527-4539.

Stromeyer CF 3rd, Kranda K, Sternheim CE (1978) Selective chromatic adaptation at different spatial frequencies. Vision Res 18:427-437.

Stromeyer CF 3rd, Chaparro A, Rodriguez C, Chen D, Hu E, Kronauer RE (1998) Short-wave cone signal in the red-green detection mechanism. Vision Res 38:813-826.

Sun H, Smithson HE, Zaidi Q, Lee BB (2006) Specificity of cone inputs to macaque retinal ganglion cells. J Neurophysiol 95:837-849.

Szmajda BA, Buzás P, Fitzgibbon T, Martin PR (2006) Geniculocortical relay of blue-off signals in the primate visual system. Proc Natl Acad Sci USA 103:19512-19517.

Tailby C, Solomon SG, Lennie P (2008a) Functional asymmetries in visual pathways carrying S-cone signals in macaque. J Neurosci 28:4078-4087.

Tailby C, Szmajda BA, Buzás P, Lee BB, Martin PR (2008b) Transmission of blue $(S)$ cone signals through the primate lateral geniculate nucleus. J Physiol 586:5947-5967.

Tsukamoto Y, Omi N (2015) OFF bipolar cells in macaque retina: typespecific connectivity in the outer and inner synaptic layers. Front Neuroanat 9:122.

Valberg A, Lee BB, Tigwell DA (1986) Neurones with strong inhibitory s-cone inputs in the macaque lateral geniculate nucleus. Vision Res 26: 1061-1064.

Vassilev A, Mihaylova MS, Racheva K, Zlatkova M, Anderson RS (2003) Spatial summation of S-cone ON and OFF signals: effects of retinal eccentricity. Vision Res 43:2875-2884.

Watanabe M, Rodieck RW (1989) Parasol and midget ganglion cells of the primate retina. J Comp Neurol 289:434-454.

Wool LE, Komban SJ, Kremkow J, Jansen M, Li X, Alonso J-M, Zaidi Q (2015) Salience of unique hues and implications for color theory. J Vis 15(2):10, 1-11.

Wool LE, Crook JD, Troy JB, Packer OS, Zaidi Q, Dacey DM (2018) Nonselective wiring accounts for red-green opponency in midget ganglion cells of the primate retina. J Neurosci 38:1520-1540.

Zlatkova MB, Vassilev A, Anderson RS (2008) Resolution acuity for equiluminant gratings of S-cone positive or negative contrast in human vision. J Vis $8(3): 9,1-10$. 